\title{
The Extended Rigid Body and the Pendulum Revisited
}

\author{
M. de la Cruz, N. Gaspar, R. Linares
}

In this paper we revisit the construction by which the $S L(2, \mathbb{R})$ symmetry of the Euler equations allows a simple pendulum to be obtained from a rigid body. We begin by reviewing the original relation found by Holm and Marsden in which, starting from the two integrals of motion of the extended rigid body with Lie algebra iso(2) and introducing a proper momentum map, it is possible to obtain both the Hamiltonian and the equations of motion of the pendulum. Important in this construction is the fact that both integrals of motion have the geometry of an elliptic cylinder. By considering the whole $S L(2, \mathbb{R})$ symmetry group, in this contribution we give all possible combinations of the integrals of motion and the corresponding momentum maps that produce the simple pendulum, showing that this system can also appear when the geometry of one of the integrals of motion is given by a hyperbolic cylinder and the other by an elliptic cylinder. As a result, we show that, from the extended rigid body with Lie algebra iso $(1,1)$, it is possible to obtain the pendulum, but only in circulating movement. Finally, as a byproduct of our analysis we provide the momentum maps that give origin to the pendulum with an imaginary time. Our discussion covers both the algebraic and the geometric point of view.

Keywords: free motion of a rigid body, simple pendulum, bi-Hamiltonian structures, momentum maps, symplectic reduction

Received December 11, 2019

Accepted March 20, 2020

The work of M de la $\mathrm{C}$ and NG is supported by the Ph.D. scholarship program of the Universidad Autónoma Metropolitana. The work of RL is partially supported from CONACyT Grant No. 237351.

Manuel de la Cruz López

fisikito@xanum.uam.mx

Néstor de Jésus Gaspar Rodríguez

g.r.nestor@outlook.com

Román Linares Romero

lirr@xanum.uam.mx

Departamento de Física, Universidad Autónoma Metropolitana Iztapalapa

San Rafael Atlixco 186, 09340, México City, México

RUSSIAN JOURNAL OF NONLINEAR DYNAMICS, 2020, 16(1), 133-159 


\section{Introduction}

The simple pendulum and the torque-free rigid body are two well-understood physical systems in both classical and quantum mechanics. The first systematic study of the pendulum is attributed to Galileo Galilei around 1602 and its dynamical description culminated with the development of the elliptic functions by Abel [16] and Jacobi [1, 17], which turn out to be the analytical solutions to the equation of motion of the pendulum (for a review of elliptic functions see, for instance, $[2-7]$, and [18-20] for the solutions of the pendulum). The quantization of the pendulum is based on the equivalence between the Schrödinger equation and the Mathieu differential equation, a result developed originally by Condon in 1928 [21] and a source of subsequent analysis of different aspects of the quantum system [22, 23]. On the other hand, in 1758 Euler showed that the equations of motion that describe the rotation of a rigid body form a set of vectorial quasi-linear first-order ordinary differential equations [24]. A geometric construction of the solution was given later on by Poinsot [8], and analytically these solutions are given, as for the simple pendulum, by elliptic functions (see, for instance, [9-12] and references therein). The quantization of the problem was attacked first by Kramers and Ittmann [25] and since then many authors have contributed to a deeper understanding of many aspects of the problem [15, 26-32].

Despite the old age of these problems, from time to time there are some new physical aspects uncovered about these systems that contribute to our knowledge and understanding of physics in general. The list is long and here we point out just four examples: i) In 1973 Y. Nambu, taking the Liouville theorem as a guiding principle, proposed a generalization of the classical Hamiltonian dynamics by superseding the usual two-dimensional phase space with an $n$-dimensional one [33]. The dynamics in the new phase space was formulated via an $n$-linear fully antisymmetric Nambu bracket with two or more "Hamiltonians"; as an example, Nambu applied his formalism to the free rigid body. ii) Motivated by an article by Deprit [34] and using the $S L(2, \mathbb{R})$ symmetry of the Euler equations, in 1991 Holm and Marsden built a new Hamiltonian in such a way that the new dynamical system, known in the literature as an extended rigid body, can be written as a Lie-Poisson system whose different Lie algebras structures can be $\mathfrak{s o}(3), \mathfrak{i s o}(2)$ or $\mathfrak{h e i s}_{3}$ [35]; particularly interesting is the $\mathfrak{i s o}(2)$ case, where the phase space of the Eulerian top is filled with invariant elliptic cylinders, on each of which the dynamics, in elliptic coordinates, is the dynamics of a standard simple pendulum. iii) In 1995 R. Montgomery computed the change in the geometric phase for the attitude of the rigid body when the angular momentum vector in the body frame performs one period of its motion [36]. iv) Finally, in 2017 [37] the free rotation of a classical rigid body was used in the control of two-level quantum systems by means of external electromagnetic pulses. In particular, the authors showed that the dynamics of a rigid body can be used to implement one-qubit quantum gates.

In this paper we are interested in exploring deeper the relation between the extended rigid body and the simple pendulum. As we have mentioned above, in the original paper [35] Holm and Marsden showed that the different Lie algebras structures of the extended rigid body are $\mathfrak{s o}(3), \mathfrak{i s o}(2)$ or $\mathfrak{h e i s}_{3}$, however, in [38] the authors showed that the complete list of possible Lie algebras includes all those related to $\mathfrak{s o}(3)$ via analytical continuation and group contractions, which means that the algebra iso $(1,1)$ must be also included. Even more, according to [35], the pendulum can be obtained from the extended rigid body if, from the geometrical point of view, the level surfaces of the two new integrals of motion have the shape of elliptic cylinders, which leads to the fact that the corresponding Lie algebra is $\mathfrak{i s o}(2)$. Very recently, using a representation of the rigid body in terms of two free parameters $e_{0}$ and $\kappa$ [39] instead of the usual five parameters (energy $E$, magnitude of the angular momentum $L$, and the three principal moments 
of inertia $I_{1}, I_{2}$ and $\left.I_{3}\right)$, the classification of the inequivalent $S L(2, \mathbb{R})$ combinations of the integrals of motion was discussed both algebraically and geometrically. It turns out that, whereas the geometry of the integral of motion that represents the square of the angular momentum continues being an $S^{2}$ sphere, the geometry of the integral of motion associated to the kinetic energy which is an ellipsoid in the five parameters representation of the rigid body, is replaced by an elliptic hyperboloid that can be either of one or two sheets depending on the numerical values of both $e_{0}$ and $\kappa$, making the classification of the $S L(2, \mathbb{R})$ combinations of the integrals of motion richer. A result of this paper is to show that, considering all possible different geometries of the new integrals of motion, it is possible to obtain the pendulum also when the geometries associated to them are an elliptic cylinder and a hyperbolic cylinder. Specifically, when the cotangent space of the simple pendulum is given by the elliptic cylinder, the Lie algebra of the Hamiltonian vector fields associated to the coordinates in the rigid body-fixed frame is iso( 2$)$, whereas if the cotangent space is given by the hyperbolic cylinder, the Lie algebra is iso $(1,1)$. Even more, we show explicitly that for the iso(2) case we always obtain the whole set of motions of the pendulum, whereas for the $\mathfrak{i s o}(1,1)$ case we get the circulating motions, but not the oscillatory ones. To the best of our knowledge this result has not been discussed previously in the literature.

Our exposition is as self-contained as possible. Section 2 is dedicated to summarizing the main characteristics of the rigid body and its equations of motion, especially the $S L(2, \mathbb{R})$ symmetry of the latter. In Section 3 we discuss the Hamiltonian structure of the simple pendulum as a function of both a real time and an imaginary time. The study of the whole $S L(2, \mathbb{R})$ transformations can be divided into three general sets where each set contains different fixed forms of the $S L(2, \mathbb{R})$ matrices. One of the three sets does not give origin to the pendulum and therefore we focus on the other two. Section 4 is devoted to the set of matrices where the relation between the rigid body and the simple pendulum can be established. This set can be subdivided into two general cases determined by the geometry of the integrals of motion, Section 4.1 is dedicated to the case where both integrals of motion are given geometrically by elliptic cylinders, whereas Sections 4.2 and 4.3 are devoted to the study of the cases where one integral of motion has the geometry of an elliptic cylinder and the other integral of motion has the geometry of a hyperbolic cylinder. In Section 5 we discuss the third and last set; as we will argue, this set is a limiting case of the one in Section 4 and therefore there does also exist a relation between the simple pendulum and the rigid body. Our conclusions are given in Section 6 .

\section{The Euler equations and its $S L(2, \mathbb{R})$ symmetry}

The torque-free rigid body motion is one of the best understood systems in physics and the number of papers and books discussing their dynamical properties is overwhelming. However, there are still some properties related to this system that deserve to be explored further. In this section we give a short summary of the characteristics of the system that are relevant for our analysis of the relation between the so-called extended rigid body and the simple pendulum. We base our discussion on $[10,11,35,39]$.

\subsection{The Euler equations and the integrals of motion}

It is well known that in a body-fixed reference frame the motion of the rigid body is governed by the Euler equations (see, for instance, [10])

$$
\frac{d \vec{L}}{d t}=\vec{L} \times I^{-1} \vec{L}
$$

RUSSIAN JOURNAL OF NONLINEAR DYNAMICS, 2020, 16(1), 133-159 
where $\vec{L}$ is the vector of angular momentum and $I$ is the moment of inertia tensor. If the bodyfixed frame is oriented to coincide with the principal axes of inertia, the tensor $I$ is diagonal. Without loss of generality, in the following discussion we assume that the principal moments of inertia satisfy the inequality

$$
I_{1}<I_{2}<I_{3} .
$$

When written in a basis, the $L_{i}$ components of the angular momentum are the generators of a $\mathfrak{s o}(3)$ Lie algebra

$$
\left[L_{i}, L_{j}\right]=\varepsilon_{i j k} L_{k}
$$

The system has two integrals of motion, the rotational kinetic energy $C_{1}$ and the square of the angular momentum $C_{2}$, which are given in terms of the moments of inertia and the components of $\vec{L}$ as

$$
C_{1}\left(L_{1}, L_{2}, L_{3}\right) \equiv \frac{L_{1}^{2}}{2 I_{1}}+\frac{L_{2}^{2}}{2 I_{2}}+\frac{L_{3}^{2}}{2 I_{3}}
$$

and

$$
C_{2}\left(L_{1}, L_{2}, L_{3}\right) \equiv L_{1}^{2}+L_{2}^{2}+L_{3}^{2},
$$

respectively. The dynamical problem is usually solved using the Poinsot construction, in an Euclidean space $\mathbb{R}^{3}$ whose coordinates are the components of the angular momentum. When the vector $\vec{L}$ moves relative to the axes of inertia of the top, it lies along the curve of intersection of the surfaces $C_{1}=E=$ constant (an ellipsoid with semiaxes $\sqrt{2 E I_{1}}, \sqrt{2 E I_{2}}$ and $\sqrt{2 E I_{3}}$ ) and $C_{2}=L^{2}=$ constant (a sphere of radius $L$ ). Because $2 E I_{1}<L^{2}<2 E I_{3}$, the radius of the sphere has a value between the minimum and maximum values of the semiaxes of the ellipsoid. At first sight the solutions of the Euler equations (2.1) depend on five different parameters, the three moments of inertia, the energy $E$ and the square of the angular momentum $L^{2}$. However, it has been shown that the problem can be rewritten in such a way that the solutions depend only on two parameters $[11,25,28,30]$. The first parameter $e_{0}$ is related to the quotient $E / L^{2}$, whereas the second parameter $\kappa$ codifies the values of the three moments of inertia. Specifically, if $\mathcal{I}$ is the diagonal matrix: $\mathcal{I}=\operatorname{diag}\left(1 / I_{1}, 1 / I_{2}, 1 / I_{3}\right)$, we can establish, in a first step, a relation between the five parameters: $\left\{I_{i}\right\}, E$ and $L^{2}$ with the four parameters: $\left\{e_{i}\right\}$ and $e_{0}$, through the equations

$$
\frac{1}{I_{i}}-\frac{1}{3} \operatorname{Tr} \mathcal{I} \equiv \sqrt{\frac{g_{2}}{3}} e_{i} \quad \frac{2 E}{L^{2}}-\frac{1}{3} \operatorname{Tr} \mathcal{I} \equiv \sqrt{\frac{g_{2}}{3}} e_{0}
$$

with $i=1,2,3$ and

$$
g_{2} \equiv \frac{2}{3}\left[\left(\frac{1}{I_{1}}-\frac{1}{I_{2}}\right)^{2}+\left(\frac{1}{I_{2}}-\frac{1}{I_{3}}\right)^{2}+\left(\frac{1}{I_{3}}-\frac{1}{I_{1}}\right)^{2}\right]>0 .
$$

Notice that the new four parameters are dimensionless, and, abusing the language, we can call the set $\left\{e_{i}\right\}$ dimensionless inertia parameters; analogously, although $e_{0}$ is not the energy of the system, we can call it the dimensionless energy parameter. The dimensionless inertia parameters can be written in terms of only one angular parameter $\kappa$ in the form

$$
e_{1}=\cos (\kappa), \quad e_{2}=\cos (\kappa-2 \pi / 3), \quad e_{3}=\cos (\kappa+2 \pi / 3),
$$

because they are restricted to satisfy the conditions

$$
e_{1}+e_{2}+e_{3}=0, \quad e_{1}^{2}+e_{2}^{2}+e_{3}^{2}=3 / 2 \quad \text { and } \quad e_{1} e_{2} e_{3}=(\cos 3 \kappa) / 4 .
$$


Geometrically, in the three-dimensional space $\left\{e_{1}, e_{2}, e_{3}\right\}$, the first condition in (2.9) represents a plane crossing the origin and the second condition represents a sphere of radius $\sqrt{3 / 2}$. The intersection of these two surfaces is a circle which is parameterized by the angular parameter $\kappa$. The role of $g_{2}$ (Eq. (2.7)) is to change the size of the sphere and therefore the size of the intersecting circle. It is clear that, given a specific rigid body or equivalently a set of values for the three principal moments of inertia $I_{i}$, the value of the angular parameter $\kappa$ is completely determined.

In general $\kappa \in[0,2 \pi]$, but notice that condition (2.2) in terms of the dimensionless inertia parameters is obtained if the free parameter $\kappa$ takes values in the subinterval $\kappa \in(0, \pi / 3)$, i.e.,

$$
e_{3}<e_{2}<e_{1}
$$

The other five subintervals of length $\pi / 3$ produce the other five possible orders for the $e_{i}$ 's, for instance, if $\kappa \in(\pi / 3,2 \pi / 3)$ the parameters ordering is $e_{3}<e_{1}<e_{2}$, etc. For any value of $\kappa$, at least one of the inertia parameters is positive, another is negative and the third may be either positive, zero or negative. Throughout the paper we work in the interval $\kappa \in(0, \pi / 3)$, for which $e_{1}>0, e_{3}<0$ and $e_{2}$ can be either positive, negative or zero.

Finally, introducing the dimensionless coordinates $u_{i} \equiv L_{i} / L$, the Euler equations (2.1) are rewritten as

$$
\frac{d u_{i}}{d t}=\frac{1}{2} \sqrt{\frac{g_{2}}{3}} L \varepsilon_{i j k}\left(e_{k}-e_{j}\right) u_{j} u_{k}
$$

where we are using the Einstein summation convention. It is possible to absorb the factor $\sqrt{\frac{g_{2}}{3}} L$ by defining a dimensionless time parameter: $x \equiv t \sqrt{\frac{g_{2}}{3}} L$ to obtain

$$
\dot{u}_{i}=\frac{1}{2} \varepsilon_{i j k}\left(e_{k}-e_{j}\right) u_{j} u_{k},
$$

where $(\cdot)$ denotes the derivative with respect to dimensionless time $x$. In a similar fashion, the integrals of motion (2.4) and (2.5) read now as

$$
\begin{aligned}
& C_{1}\left(u_{1}, u_{2}, u_{3}\right) \equiv e_{1} u_{1}^{2}+e_{2} u_{2}^{2}+e_{3} u_{3}^{2}, \\
& C_{2}\left(u_{1}, u_{2}, u_{3}\right) \equiv u_{1}^{2}+u_{2}^{2}+u_{3}^{2} .
\end{aligned}
$$

In the dimensionless angular momentum space $\mathbb{R}^{3}:\left(u_{1}, u_{2}, u_{3}\right), C_{2}=1$ and it represents a unitary sphere, whereas for the other integral: $C_{1}=e_{0}$ and the ellipsoid (2.4) is replaced by an elliptic hyperboloid that can be either of one or two sheets depending on the numerical values of both $2 E / L^{2}$ and $\kappa$, or equivalently on the number of positive and negative coefficients (dimensionless inertia parameters) in the equation. In this latter case the level surface of the integral of motion can also have the geometry of an elliptic cone or a hyperbolic cylinder in the proper limit situations. A complete classification of the geometrical shapes of the level surface of the integral of motion $C_{1}$ can be found in [39].

Regarding the solutions of the Euler equations (2.12), given the ordering (2.10) of the dimensionless inertia parameters, the solutions depend on the relative value between $e_{0}$ and $e_{2}$. Here we are not going into detail, but for purposes of completeness in our exposition, we present the explicit solutions. Details can be found, for instance, in [11]. 
I. Case $e_{3}<e_{2}<e_{0}<e_{1} \quad$ (i.e. $\left.1 / I_{2}<2 E / L^{2}\right)$.

When the energy parameter $e_{0}$ is between the inertia parameters $e_{2}$ and $e_{1}$, the solutions are given by

$$
\begin{aligned}
& u_{1}(\tau)=\operatorname{sn}\left(\tau^{\prime}, m_{c}\right) \operatorname{dn}(\tau, m), \quad u_{2}(\tau)=\operatorname{dn}\left(\tau^{\prime}, m_{c}\right) \operatorname{sn}(\tau, m), \\
& u_{3}(\tau)=\operatorname{cn}\left(\tau^{\prime}, m_{c}\right) \operatorname{cn}(\tau, m),
\end{aligned}
$$

where $\tau$ is a dimensionless time parameter defined as

$$
\tau=x \sqrt{\left(e_{1}-e_{2}\right)\left(e_{0}-e_{3}\right)} .
$$

Here the amplitudes of the solutions are written as Jacobi elliptic functions with parameter $\tau^{\prime}=$ constant and are related to the dimensionless parameters $e_{i}$ and $e_{0}$ by

$$
\operatorname{sn}^{2}\left(\tau^{\prime}, m_{c}\right)=\frac{e_{0}-e_{3}}{e_{1}-e_{3}}, \quad \operatorname{cn}^{2}\left(\tau^{\prime}, m_{c}\right)=\frac{e_{1}-e_{0}}{e_{1}-e_{3}}, \quad \operatorname{dn}^{2}\left(\tau^{\prime}, m_{c}\right)=\frac{e_{1}-e_{0}}{e_{1}-e_{2}} .
$$

The square modulus $m$ and the complementary modulus $m_{c}$ in Eq. (2.15) are given by

$$
\begin{aligned}
& m^{2} \equiv \frac{\left(e_{2}-e_{3}\right)}{\left(e_{0}-e_{3}\right)} \frac{\left(e_{1}-e_{0}\right)}{\left(e_{1}-e_{2}\right)}=\frac{\left(e_{1}-e_{0}\right)}{\left(e_{0}-e_{3}\right)} \frac{k_{1}^{2}}{k_{2}^{2}}, \text { and } \\
& m_{c}^{2} \equiv \frac{\left(e_{0}-e_{2}\right)}{\left(e_{0}-e_{3}\right)} \frac{\left(e_{1}-e_{3}\right)}{\left(e_{1}-e_{2}\right)}=\frac{\left(e_{0}-e_{2}\right)}{\left(e_{0}-e_{3}\right)} \frac{1}{k_{2}^{2}},
\end{aligned}
$$

which take values in the interval $0<m^{2}<1$ and $0<m_{c}^{2}<1$ and satisfy $m^{2}+m_{c}^{2}=1$. Here the quotients

$$
k_{1}^{2}=\frac{e_{2}-e_{3}}{e_{1}-e_{3}} \quad \text { and } \quad k_{2}^{2}=\frac{e_{1}-e_{2}}{e_{1}-e_{3}}
$$

satisfy in a similar way $k_{1}^{2}+k_{2}^{2}=1$.

II. Case $e_{3}<e_{0}<e_{2}<e_{1} \quad$ (i.e., $\left.2 E / L^{2}<1 / I_{2}\right)$.

In this case the solutions are given by

$$
\begin{aligned}
& u_{1}=\operatorname{cn}\left(\tau^{\prime}, i \frac{m_{c}}{m}\right) \operatorname{cn}\left(m \tau, \frac{1}{m}\right), \quad u_{2}=\operatorname{dn}\left(\tau^{\prime}, i \frac{m_{c}}{m}\right) \operatorname{sn}\left(m \tau, \frac{1}{m}\right), \\
& u_{3}=\operatorname{sn}\left(\tau^{\prime}, i \frac{m_{c}}{m}\right) \operatorname{dn}\left(m \tau, \frac{1}{m}\right),
\end{aligned}
$$

where the square modulus of the elliptic function takes values in the interval $0<1 / m^{2}<1$, with $m^{2}$ as defined in (2.18), but due to the fact that $e_{3}<e_{0}<e_{2}<e_{1}, m^{2}>1$. The time parameter $\tau$ is the one defined in (2.16), whereas the amplitudes are written as Jacobi elliptic functions at parameter $\tau^{\prime}=$ constant

$$
\begin{aligned}
\operatorname{sn}^{2}\left(\tau^{\prime}, i \frac{m_{c}}{m}\right) & =\frac{e_{1}-e_{0}}{e_{1}-e_{3}}, \quad \operatorname{cn}^{2}\left(\tau^{\prime}, i \frac{m_{c}}{m}\right)=\frac{e_{0}-e_{3}}{e_{1}-e_{3}}, \\
\operatorname{dn}^{2}\left(\tau^{\prime}, i \frac{m_{c}}{m}\right) & =\frac{e_{0}-e_{3}}{e_{2}-e_{3}} .
\end{aligned}
$$


The physical interpretation of the solutions is straightforward, the curves (2.15) and (2.20) are the parameterization of the intersection of the unitary sphere of angular momentum and the corresponding surface of the integral of motion (2.13) [39] (see Fig. 1).

At this point it is convenient to make clear the possible values that can take the square modulus $m^{2}$, the complementary modulus $m_{c}^{2}$, their inverse values and the quotients of these quantities. The reason for it is that these quantities will appear when the connection between the rigid body and the simple pendulum is established.

Table 1. Intervals of values of the square modulus $m^{2}$, complementary modulus $m_{c}^{2}$, their inverse values $1 / m^{2}, 1 / m_{c}^{2}$ and the quotients $m^{2} / m_{c}^{2}$ and $m_{c}^{2} / m^{2}$, as functions of the relative values between the dimensionless energy parameter $e_{0}$ and the dimensionless inertia parameter $e_{2}$

\begin{tabular}{|c|c|c|c|c|c|c|}
\hline & $m^{2}$ & $m_{c}^{2}$ & $\frac{1}{m^{2}}$ & $-\frac{m_{c}^{2}}{m^{2}}$ & $\frac{1}{m_{c}^{2}}$ & $-\frac{m^{2}}{m_{c}^{2}}$ \\
\hline$e_{2}<e_{0}$ & $(0,1)$ & $(0,1)$ & $(1, \infty)$ & $(-\infty, 0)$ & $(1, \infty)$ & $(-\infty, 0)$ \\
$e_{0}<e_{2}$ & $(1, \infty)$ & $(-\infty, 0)$ & $(0,1)$ & $(0,1)$ & $(-\infty, 0)$ & $(1, \infty)$ \\
\hline
\end{tabular}

Notice that, just as $m^{2}$ and $m_{c}^{2}$ are complementary to each other in the sense that $m^{2}+m_{c}^{2}=1$, so the couple $1 / m^{2}$ and $-m_{c}^{2} / m^{2}$ are complementary to each other, as well as the couple $1 / m_{c}^{2}$ and $-m^{2} / m_{c}^{2}$, i.e.,

$$
m^{2}+m_{c}^{2}=1, \quad \frac{1}{m^{2}}-\frac{m_{c}^{2}}{m^{2}}=1, \quad \frac{1}{m_{c}^{2}}-\frac{m^{2}}{m_{c}^{2}}=1 .
$$

\section{2. $S L(2, \mathbb{R})$ symmetries of the Euler equations}

The rigid body problem has two integrals of motion and it is completely integrable. It constitutes an example of a bi-Hamiltonian system which admits two Hamiltonian descriptions with respect to two compatible Poisson structures on a Poisson manifold [10]. In order to make manifest the gauge symmetries of the Euler equations, we notice that its dimensionless form

$$
\dot{\vec{u}}=\vec{u} \times \epsilon \vec{u}
$$

with $\epsilon$ a diagonal matrix of the form $\epsilon=\operatorname{diag}\left(e_{1}, e_{2}, e_{3}\right)$ can be rewritten as the gradient of two scalar functions

$$
\dot{\vec{u}}=\nabla l \times \nabla h,
$$

where

$$
\begin{gathered}
h \equiv \frac{1}{2} C_{1}\left(u_{1}, u_{2}, u_{3}\right)-\frac{1}{2} e_{0}=0 \Rightarrow \epsilon \vec{u}=\nabla h=\left(e_{1} u_{1}, e_{2} u_{2}, e_{3} u_{3}\right), \\
l \equiv \frac{1}{2} C_{2}\left(u_{1}, u_{2}, u_{3}\right)-\frac{1}{2}=0 \Rightarrow \vec{u}=\nabla l=\left(u_{1}, u_{2}, u_{3}\right) .
\end{gathered}
$$

This form of writing the Euler equations makes explicit its invariance under any $S L(2, \mathbb{R})$ transformation [35]. It is straightforward to check that the transformation

$$
\left(\begin{array}{l}
\mathcal{H} \\
\mathcal{N}
\end{array}\right)=\left(\begin{array}{ll}
a & b \\
c & d
\end{array}\right)\left(\begin{array}{l}
h \\
l
\end{array}\right)
$$


with $a d-b c=1$ leads to

$$
\dot{\vec{u}}=\nabla \mathcal{N} \times \nabla \mathcal{H}
$$

At this point we can consider either of the new integrals of motion $\mathcal{N}$ or $\mathcal{H}$ as the Hamiltonian surface, i.e., the property that the system is bi-Hamiltonian has been maintained. If we choose the surface $\mathcal{H}$ as the Hamiltonian, we have a dynamical system with Hamiltonian $H$, and any dynamical quantity $Q$ evolves with time according to

$$
\dot{Q}=X_{\mathcal{H}} Q
$$

where the generator $X_{G}$ is given by

$$
X_{G}=(\nabla \mathcal{N} \times \nabla G) \cdot \nabla .
$$

Notice that we use different letters to denote the Hamiltonian or integral of motion: $H=H\left(u_{1}, u_{2}, u_{3}\right)$ and the level surface of the integral of motion: $\mathcal{H}\left(u_{1}, u_{2}, u_{3}\right) \equiv H\left(u_{1}, u_{2}, u_{3}\right)-$ $-E=0$.

According to the transformation (2.27), the generator $X_{G}$ in components form is expressed as

$$
\begin{aligned}
X_{G} & =\left(c e_{1}+d\right) u_{1}\left(\frac{\partial G}{\partial u_{2}} \frac{\partial}{\partial u_{3}}-\frac{\partial G}{\partial u_{3}} \frac{\partial}{\partial u_{2}}\right)+\left(c e_{2}+d\right) u_{2}\left(\frac{\partial G}{\partial u_{3}} \frac{\partial}{\partial u_{1}}-\frac{\partial G}{\partial u_{1}} \frac{\partial}{\partial u_{3}}\right)+ \\
& +\left(c e_{3}+d\right) u_{3}\left(\frac{\partial G}{\partial u_{1}} \frac{\partial}{\partial u_{2}}-\frac{\partial G}{\partial u_{2}} \frac{\partial}{\partial u_{1}}\right) .
\end{aligned}
$$

In order to determine the structure of the Lie algebra, we calculate the Lie-Poisson brackets for the Hamiltonian vector fields associated with the coordinate functions $u_{i}$

$$
\begin{aligned}
& X_{u_{1}}=\left(c e_{3}+d\right) u_{3} \partial_{2}-\left(c e_{2}+d\right) u_{2} \partial_{3}, \\
& X_{u_{2}}=\left(c e_{1}+d\right) u_{1} \partial_{3}-\left(c e_{3}+d\right) u_{3} \partial_{1}, \\
& X_{u_{3}}=\left(c e_{2}+d\right) u_{2} \partial_{1}-\left(c e_{1}+d\right) u_{1} \partial_{2} .
\end{aligned}
$$

Explicitly, the Lie-Poisson brackets are given by

$$
\begin{aligned}
& {\left[X_{u_{1}}, X_{u_{2}}\right]=\left(c e_{3}+d\right) X_{u_{3}},} \\
& {\left[X_{u_{2}}, X_{u_{3}}\right]=\left(c e_{1}+d\right) X_{u_{1}},} \\
& {\left[X_{u_{3}}, X_{u_{1}}\right]=\left(c e_{2}+d\right) X_{u_{2}} .}
\end{aligned}
$$

Regarding the classification of the different Lie algebras that arise from the $\mathfrak{s l}(2, \mathbb{R})$ invariant orbits, it turns out that, in addition to the $\mathfrak{s o}(3)$ Lie algebra (2.3) which corresponds to the rigid body, the algebras $\mathfrak{s o}(2,1), \mathfrak{i s o}(2), \mathfrak{i s o}(1,1)$ and $\mathfrak{h e i}_{3}$, also emerge. The systems corresponding to these algebras are termed extended rigid bodies. The whole classification can be found, for instance, in [39].

Because it is relevant for our discussion, it is important to emphasize that, if instead we take the level surface of the integral of motion $\mathcal{N}$ as the Hamiltonian surface, then the generator $X_{G}$ is given by

$$
X_{G}=-(\nabla \mathcal{H} \times \nabla G) \cdot \nabla \quad \Rightarrow \quad \dot{Q}=X_{\mathcal{N}} Q .
$$


Clearly, $X_{\mathcal{N}}=-X_{\mathcal{H}}$. If we let $\widetilde{X}_{u_{i}}$ denote the Hamiltonian vector fields associated to the coordinates, we obtain in this case

$$
\begin{aligned}
\widetilde{X}_{u_{1}} & =-\left(a e_{3}+b\right) u_{3} \partial_{2}+\left(a e_{2}+b\right) u_{2} \partial_{3}, \\
\widetilde{X}_{u_{2}} & =-\left(a e_{1}+b\right) u_{1} \partial_{3}+\left(a e_{3}+b\right) u_{3} \partial_{1}, \\
\widetilde{X}_{u_{3}} & =-\left(a e_{2}+b\right) u_{2} \partial_{1}+\left(a e_{1}+b\right) u_{1} \partial_{2} .
\end{aligned}
$$

With these vector fields the Lie-Poisson brackets are given explicitly by

$$
\begin{aligned}
& {\left[\widetilde{X}_{u_{1}}, \widetilde{X}_{u_{2}}\right]=-\left(a e_{3}+b\right) \widetilde{X}_{u_{3}}, \quad\left[\widetilde{X}_{u_{2}}, \widetilde{X}_{u_{3}}\right]=-\left(a e_{1}+b\right) \widetilde{X}_{u_{1}},} \\
& {\left[\widetilde{X}_{u_{3}}, \widetilde{X}_{u_{1}}\right]=-\left(a e_{2}+b\right) \widetilde{X}_{u_{2}} .}
\end{aligned}
$$

The classification of the different Lie algebras coincide with the five mentioned above. The details are completely analogous to the previous ones and we do not discuss them further.

\section{The pendulum}

As in the case of the extended rigid body, solutions of the simple pendulum system are given in terms of Jacobi elliptic functions which are defined in the whole complex plane $\mathbb{C}$ and are doubly periodic. Due to the relevance of the expressions of the pendulum energy in both real and imaginary time, for the purposes of this paper, in this section we review briefly the main characteristics of the mathematical formulation of the simple pendulum. This is a wellunderstood system and there are many interesting papers and books on the subject $[2,5,7,13$, $14,18,19,40]$. In our discussion we follow [20] mainly.

\subsection{Real time pendulum and solutions}

Let us start considering a pendulum of point mass $m$ and length $r$, in a constant downwards gravitational field, of magnitude $-g(g>0)$. If $\theta$ is the polar angle measured counterclockwise with respect to the vertical line and $\dot{\theta}$ stands for the time derivative of this angular position, the Lagrangian of the system is given by

$$
L(\theta, \dot{\theta})=\frac{1}{2} m r^{2} \dot{\theta}^{2}-m g r(1-\cos \theta) .
$$

Here the zero of the potential energy is set at the lowest vertical position of the pendulum $(\theta=2 n \pi$, with $n \in \mathbb{Z})$. The equation of motion for this system is

$$
\ddot{\theta}+\frac{g}{r} \sin \theta=0
$$

which after integration gives origin to the conservation of total mechanical energy

$$
E=\frac{1}{2} m r^{2} \dot{\theta}^{2}+2 m g r \sin ^{2}\left(\frac{\theta}{2}\right)=\text { const. }
$$

Physical solutions of Eq. (3.3) exist only if $E \geqslant 0$. We can rewrite the equation in dimensionless form, in terms of the dimensionless energy parameter: $k_{E}^{2} \equiv \frac{E}{2 m g r}$, and the dimensionless real time variable: $x \equiv \sqrt{\frac{g}{r}} t \in \mathbb{R}$ to obtain

$$
\left(\frac{p}{2}\right)^{2}+\sin ^{2}\left(\frac{\theta}{2}\right)=k_{E}^{2}
$$


where $p$ is the dimensionless angular velocity: $p(x) \equiv d \theta / d x$. By inspection of the potential we conclude that the pendulum has four different types of solutions depending on the value of the constant $k_{E}^{2}$ :

- Static equilibrium $(\dot{\theta}=0)$ : The trivial behavior occurs when either $k_{E}^{2}=0$ or $k_{E}^{2}=$ $=1$. In the first case, necessarily $\dot{\theta}=0$. For the case $k_{E}^{2}=1$ we consider also the situation where $\dot{\theta}=0$. In both cases, the pendulum does not move, it is in static equilibrium. When $\theta=2 n \pi$ the equilibrium is stable, and when $\theta=(2 n+1) \pi$ the equilibrium is unstable.

- Oscillatory motions $\left(0<k_{E}^{2}<1\right)$ : In these cases the pendulum swings to and fro, respect to a point of stable equilibrium. The analytical solutions are given by

$$
\begin{aligned}
& \theta(x)=2 \arcsin \left[k_{E} \operatorname{sn}\left(x-x_{0}, k_{E}\right)\right], \\
& p(x)=2 k_{E} \operatorname{cn}\left(x-x_{0}, k_{E}\right),
\end{aligned}
$$

where the square modulus $k^{2}$ of the elliptic functions is given directly by the energy parameter: $k^{2} \equiv k_{E}^{2}$. Here $x_{0}$ is a second constant of integration and appears when Eq. (3.4) is integrated out. It means physically that we can choose the zero of time arbitrarily. The period of the movement is $4 K$, or restoring the dimension of time, $4 K \sqrt{g / r}$, with $K$ the quarter period of the elliptic function $\operatorname{sn}\left(x-x_{0}, k_{E}\right)$.

- Asymptotical motion $\left(k_{E}^{2}=1\right.$ and $\left.\dot{\theta} \neq 0\right)$ : In this case the angle $\theta$ takes values in the open interval $(-\pi, \pi)$ and therefore, $\sin (\theta / 2) \in(-1,1)$. The particle just reaches the highest point of the circle. The analytical solutions are given by

$$
\begin{aligned}
& \theta(x)= \pm 2 \arcsin \left[\tanh \left(x-x_{0}\right)\right], \\
& p(x)= \pm 2 \operatorname{sech}\left(x-x_{0}\right) .
\end{aligned}
$$

The sign \pm corresponds to the movement from $(\mp \pi \rightarrow \pm \pi)$. Notice that $\tanh \left(x-x_{0}\right)$ takes values in the open interval $(-1,1)$ if: $x-x_{0} \in(-\infty, \infty)$. For instance, if $\theta \rightarrow \pi, x-x_{0} \rightarrow \infty$ and $\tanh \left(x-x_{0}\right)$ goes asymptotically to 1 . It is clear that this movement is not periodic. In the literature it is common to take $x_{0}=0$.

- Circulating motions $\left(k_{E}^{2}>1\right)$ : In these cases the momentum of the particle is large enough to carry it over the highest point of the circle, so that it moves round and round the circle, always in the same direction. The solutions that describe these motions are of the form

$$
\begin{aligned}
& \theta(x)= \pm 2 \operatorname{sgn}\left[\operatorname{cn}\left(k_{E}\left(x-x_{0}\right), \frac{1}{k_{E}}\right)\right] \arcsin \left[\operatorname{sn}\left(k_{E}\left(x-x_{0}\right), \frac{1}{k_{E}}\right)\right], \\
& p(x)= \pm 2 k_{E} \operatorname{dn}\left(k_{E}\left(x-x_{0}\right), \frac{1}{k_{E}}\right),
\end{aligned}
$$

where the global sign $(+)$ stands for the counterclockwise motion and the $(-)$ sign stands for the motion in the clockwise direction. The symbol $\operatorname{sgn}(x)$ stands for the piecewise sign function which we define in the form

$$
\operatorname{sgn}\left[\operatorname{cn}\left(k_{E}\left(x-x_{0}\right), \frac{1}{k_{E}}\right)\right]= \begin{cases}+1 & \text { if } \quad(4 n-1) K \leqslant k_{E}\left(x-x_{0}\right)<(4 n+1) K, \\ -1 & \text { if } \quad(4 n+1) K \leqslant k_{E}\left(x-x_{0}\right)<(4 n+3) K,\end{cases}
$$


and its role is to shorten the period of the function $\operatorname{sn}\left(k_{E}\left(x-x_{0}\right), 1 / k_{E}\right)$ by half. This fact is in agreement with the expression for $p(x)$ because the period of the elliptic function $\operatorname{dn}\left(k_{E}\left(x-x_{0}\right), 1 / k_{E}\right)$ is $2 K / k_{E}$ instead of $4 K / k_{E}$, which is the period of the elliptic function $\operatorname{sn}\left(k_{E}\left(x-x_{0}\right), 1 / k_{E}\right)$. The square modulus $k^{2}$ of the elliptic functions is equal to the inverse of the energy parameter $0<k^{2}=1 / k_{E}^{2}<1$.

These are all the possible motions of the simple pendulum. It is straightforward to check that the solutions satisfy the equation of conservation of energy (3.4) by using the following relations between the Jacobi functions (in these relations the modulus satisfies $0<k^{2}<1$ ) and its analogous relation for hyperbolic functions (which are obtained in the limit case $k=1$ )

$$
\begin{aligned}
\operatorname{sn}^{2}(x, k)+\operatorname{cn}^{2}(x, k) & =1, \\
\tanh ^{2}(x)+\operatorname{sech}^{2}(x) & =1, \\
k^{2} \operatorname{sn}^{2}(x, k)+\operatorname{dn}^{2}(x, k) & =1 .
\end{aligned}
$$

\subsection{Imaginary time pendulum}

In the analysis above, time was considered a real variable, and therefore in the solutions of the simple pendulum only the real quarter period $K$ appeared. But Jacobi elliptic functions are defined in $\mathbb{C}$ and, for instance, the function $\operatorname{sn}(z, k)$ of square modulus $0<k^{2}<1$, besides the real primitive period $4 K$, owns a pure imaginary primitive period $2 i K_{c}$, where $K$ and $K_{c}$ are the so-called quarter periods (see, for instance, [5]). In 1878 Paul Appell clarified the physical meaning of the imaginary time and the imaginary period in the oscillatory solutions of the pendulum $[7,41]$, by introducing an ingenious trick, he reversed the direction of the gravitational field: $g \rightarrow-g$, i.e., now the gravitational field is upwards. In order that the Newton equations of motion remain invariant under this change in the force, we must replace the real time variable $t$ by a purely imaginary one: $\tau \equiv \pm i t$. Making these changes in the equation of motion (3.2) leads to the equation

$$
\frac{d^{2} \theta}{d \tau^{2}}-\frac{g}{r} \sin \theta=0
$$

Writing this equation in dimensionless form requires the introduction of the pure imaginary time variable $y \equiv \pm \tau \sqrt{g / r}= \pm i x$. Integrating once the resulting dimensionless equation of motion gives origin to the following equation:

$$
\frac{1}{4}\left(\frac{d \theta}{d y}\right)^{2}-\sin ^{2}\left(\frac{\theta}{2}\right)=-k_{E}^{2}
$$

which looks like Eq. (3.4), but with an inverted potential. In order to solve this equation, we should flip the sign in the potential and rewrite the equation in such a way that it looks similar to Eq. (3.4). To achieve this aim we start by shifting the value of the potential energy by unity such that its minimum value is zero. Adding a unit of energy to both sides of the equation leads to

$$
\left(\frac{\mathbb{P}}{2}\right)^{2}+\cos ^{2}\left(\frac{\theta}{2}\right)=1-k_{E}^{2}
$$

Here $\mathbb{P}$ is the momentum as a function of imaginary time. The second step is to rewrite the potential energy in such a form that it coincides with the potential energy of (3.4), thereby 
allowing us to compare solutions. We can accomplish this by a simple translation of the graph, for instance, by translating it by angle $\pi / 2$ to the right. Defining $\theta^{\prime}=\theta-\pi$, we obtain

$$
\left(\frac{\mathbb{P}}{2}\right)^{2}+\sin ^{2}\left(\frac{\theta^{\prime}}{2}\right)=1-k_{E}^{2}
$$

It is clear that $0<1-k_{E}^{2}<1$ for oscillatory motions and $1-k_{E}^{2}<0$ for the circulating ones. It is not the purpose of this paper to review the whole set of solutions with imaginary time. For a detailed construction, the interested reader is referred, for instance, to [20].

\section{The pendulum from the extended rigid body $(c \neq 0$ and $d \neq 0)$}

In this section we review the relation between the rigid body and the simple pendulum as originally discussed by Holm and Marsden [35], and we extend it to pendulums of imaginary time. In every case we discuss the geometrical and algebraic characteristics of the relations.

A lesson learned from [35] is that, in order to have the pendulum from the rigid body it is necessary that the $S L(2, \mathbb{R})$ transformation of the integrals of motion lead to new ones that geometrically represent two perpendicular elliptic cylinders. In our parameterization, the mathematical condition for this to happen is that

$$
a e_{i}+b=0, \quad \text { and } \quad c e_{j}+d=0, \quad \text { with } \quad i \neq j .
$$

However, due to the fact that the inertia parameters are not necessarily positive, these conditions are attached not only to elliptical cylinders, but also to hyperbolic cylinders [39]. In order to have control over all different possibilities to get pendulums from the rigid body, it is necessary to list all different $S L(2, \mathbb{R})$ transformations that fulfill conditions (4.1). The transformations are divided into three general sets, where each set contains different fixed forms of the $S L(2, \mathbb{R})$ matrices. The three sets are determined by the conditions: i) $c \neq 0$ and $d \neq 0$. ii) $c \neq 0$ and $d=0$ and iii) $c=0$ and $d \neq 0[42]$. In the latter case a generic $S L((2, \mathbb{R})$ group element has the form

$$
g=\left(\begin{array}{cc}
a & b \\
0 & 1 / a
\end{array}\right), \quad \text { with } b \in \mathbb{R} .
$$

We conclude that the surface $\mathcal{N}$ is a unitary sphere and therefore in this case it is not possible to degenerate the surface to a cylinder. As a consequence, the pendulum cannot arise from group elements of this kind and we must analyze only cases i) and ii). Because the case discussed by Holm and Marsden belongs to the first set of conditions, we analyze it in this section and leave the set: $c \neq 0$ and $d=0$ for Section 5 .

The first step in our analysis is to fully classify the different cases belonging to the set $c \neq 0$ and $d \neq 0$ in which the level surfaces of the integrals of motion $\mathcal{H}$ and $\mathcal{N}$ as defined in Eq. (2.27) fulfill conditions (4.1). It is clear that there are 6 different forms to satisfy these conditions. However, these are not all independent, in fact, the difference between conditions: $a e_{i}+b=0$ and $c e_{j}+d=0$, with respect to conditions: $a e_{j}+b=0$ and $c e_{i}+d=0$, is that they interchange the role of the integrals of motion $H$ and $N$. Thus, (2.15) and (2.20) depend on the relative value of the parameter $e_{0}$ with respect to $e_{2}$, the classification of geometries for both $\mathcal{H}$ and $\mathcal{N}$ 
Table 2. Classification of the four different geometries for the integral of motion $H$, in the asymmetric extended rigid body

\begin{tabular}{|c|c|c|c|c|}
\hline Situation & $a e_{1}+b$ & $a e_{2}+b$ & $a e_{3}+b$ & $\mathcal{H}$ surface \\
\hline 1 & $=0$ & $<0$ & $<0$ & elliptic cylinder around $u_{1}$ \\
2 & $>0$ & $=0$ & $<0$ & hyperbolic cylinder around $u_{2}$ with focus on $u_{1}\left(e_{2}<e_{0}\right)$ \\
3 & $>0$ & $=0$ & $<0$ & hyperbolic cylinder around $u_{2}$ with focus on $u_{3}\left(e_{0}<e_{2}\right)$ \\
4 & $>0$ & $>0$ & $=0$ & elliptic cylinder around $u_{3}$ \\
\hline
\end{tabular}

Table 3. Classification of the four different geometries for the integral of motion $N$, in the asymmetric extended rigid body

\begin{tabular}{|c|c|c|c|c|}
\hline Situation & $c e_{1}+d$ & $c e_{2}+d$ & $c e_{3}+d$ & $\mathcal{N}$ surface \\
\hline 5 & $=0$ & $<0$ & $<0$ & elliptic cylinder around $u_{1}$ \\
6 & $>0$ & $=0$ & $<0$ & hyperbolic cylinder around $u_{2}$ with focus on $u_{1}\left(e_{2}<e_{0}\right)$ \\
7 & $>0$ & $=0$ & $<0$ & hyperbolic cylinder around $u_{2}$ with focus on $u_{3}\left(e_{0}<e_{2}\right)$ \\
8 & $>0$ & $>0$ & $=0$ & elliptic cylinder around $u_{3}$ \\
\hline
\end{tabular}

in general also depends on this relative value [39]. This fact increases the number of cases to five. We show in Table 2 the different possible geometries for the integral of motion $H$ and in Table 3 the corresponding ones for the integral of motion $N$. It is important to stress that in this classification we use the fact that the space $\left\{u_{1}, u_{2}, u_{3}\right\}$ is $\mathbb{R}^{3}$.

It is clear the five different possibilities we refer to correspond to the intersections: 1-6, 1-7, 1-8, 2-8 and 3-8. The one discussed by Holm and Marsden corresponds to the case of the intersection of two elliptical cylinders 1-8, however, we have four more possibilities which correspond to the intersection of an elliptical cylinder and a hyperbolic cylinder. For the best of our knowledge, these four cases have not been discussed in the literature; one of the aims of this section is to give them an interpretation as pendulums. At this point it is convenient to stress the two main differences between our analysis and the original discussion in [35]. 1) The discussion of Holm and Marsden was given using the moments of inertia (2.2) as parameters, whereas here we use a different parameterization, the one in terms of the dimensionless inertia parameters $\left\{e_{i}\right\}$, which produce slight differences in the discussion. 2) In addition to the original momentum map $\left\{u_{i}\right\} \rightarrow\left\{u_{i}(\theta, p)\right\}$ whose main characteristic is to have a real pendulum momentum $p$ or equivalently a real angular coordinate $\theta$ and a real time $t$, in our discussion we will also consider momentum maps where the real pendulum momentum $p$ is rewritten as $p=i \mathbb{P}$ with the new momentum: $\mathbb{P}=-i p$ purely imaginary. As discussed in subsection 3.2, this momentum is a consequence of introducing a purely imaginary time. When time is real the final dimensionless angular momentum space remains $\mathbb{R}^{3}$, whereas the associated pendulum phase space is $\mathbb{R} \times S^{1}$. When time is purely imaginary, the final angular momentum space is $\mathbb{R}^{2} \times i \mathbb{R}$, whereas the pendulum phase space is built with a real coordinate $\theta$ and a purely imaginary momentum $\mathbb{P}$. We start discussing the original case of Holm and Marsden (1-8). 


\subsection{Intersection of two elliptic cylinders}

Choosing the $a, b, c$ and $d$ nonvanishing values of the group element $g \in S L(2, \mathbb{R})$ to satisfy

$$
a e_{1}+b=c e_{3}+d=0,
$$

fix two of the three free parameters of $g$. Substituting $b$ and $d$ into the expressions (2.27) for the level surfaces of the integrals of motion, we obtain

$$
\begin{array}{rlrl}
\mathcal{H}: & -\frac{a}{2}\left(e_{1}-e_{2}\right) u_{2}^{2}-\frac{a}{2}\left(e_{1}-e_{3}\right) u_{3}^{2} & =-\frac{a}{2}\left(e_{1}-e_{0}\right), \\
\mathcal{N}: & \frac{c}{2}\left(e_{1}-e_{3}\right) u_{1}^{2}+\frac{c}{2}\left(e_{2}-e_{3}\right) u_{2}^{2}=\frac{c}{2}\left(e_{0}-e_{3}\right) .
\end{array}
$$

Notice that the surfaces do not depend on the specific values of both $a$ and $c$. However, we can fix one of these parameters in terms of the other using the condition $a d-b c=1$, which in this case produce the condition $a c=1 /\left(e_{1}-e_{3}\right)$. In other words, Holm and Marsden considered an element $g \in S L(2, \mathbb{R})$ of the type

$$
g=\left(\begin{array}{cc}
\frac{1}{c\left(e_{1}-e_{3}\right)} & -\frac{e_{1}}{c\left(e_{1}-e_{3}\right)} \\
c & -c e_{3}
\end{array}\right),
$$

which depends only on one free parameter $c \neq 0$. Originally this parameter was fixed to the value $c=1$, although it is not necessary to fix it because the $S L(2, \mathbb{R})$ combination of $h$ and $l$ leads to expressions that do not depend on $c$. It is clear that, given the ordering (2.10), the coefficients in (4.6) have the property $a>0, b<0$ and $d>0$.

The equations that determine the level surfaces of the integrals of motion can be written finally as

$$
\begin{aligned}
& \mathcal{H}: \quad k_{2}^{2} u_{2}^{2}+u_{3}^{2}=\frac{e_{1}-e_{0}}{e_{1}-e_{3}}, \\
& \mathcal{N}: \quad u_{1}^{2}+k_{1}^{2} u_{2}^{2}=\frac{e_{0}-e_{3}}{e_{1}-e_{3}},
\end{aligned}
$$

which generically represent two elliptic cylinders, $\mathcal{H}$ with axis on cordinate $u_{1}$ and $\mathcal{N}$ with axis along $u_{3}$. Here $k_{1}^{2}$ and $k_{2}^{2}$ are the ones defined in (2.19). At this point the transverse sections of the cylinders are ellipses whose semimajor and semiminor axes depend on the value of the parameter $e_{0}$, i.e., they have variable size and the intersections are exactly the same as the ones in Fig. 1a because the $S L(2, \mathbb{R})$ transformations change the geometry of the integrals of motion, but leave invariant the intersections [33].

The strategy is now to make a change of variables in such a way that one of the cylinders becomes circular using only trigonometric functions. Once this aim is achieved, the second cylinder cannot be mapped to a circular cylinder simultaneously without introducing elliptic functions. This strategy can be applied in two different ways, in one case $\mathcal{H}$ becomes the circular cylinder, whereas in the second case the integral whose geometry becomes the circular cylinder is $\mathcal{N}$. Interestingly, these two cases produce the same physical system as expected for a bi-Hamiltonian system, although the physical origin differs in both cases as we will explain. The change of variables or technically the momentum maps we implement [10] are analogous to the one performed by Holm and Marsden. As a result of the mapping one of the resulting integrals of motion have the form of the Hamiltonian of the simple pendulum and we can identify directly if the energy produces an oscillating or a circulating movement. Additionally, we also introduce momentum maps for imaginary time. 
- Simple pendulum with real time and Hamiltonian $\mathcal{H}$ : Consider the momentum map

$$
u_{1} \equiv \sqrt{\frac{e_{0}-e_{3}}{e_{1}-e_{3}}} \cos \left(\frac{\theta}{2}\right), \quad u_{2} \equiv \frac{1}{k_{1}} \sqrt{\frac{e_{0}-e_{3}}{e_{1}-e_{3}}} \sin \left(\frac{\theta}{2}\right), \quad u_{3} \equiv \frac{k_{2}}{k_{1}} \sqrt{\frac{e_{0}-e_{3}}{e_{1}-e_{3}}} \frac{p}{2} .
$$

The expression for the level surface of the integral of motion $\mathcal{N}$ is explicitly satisfied, whereas the expression for the integral of motion $\mathcal{H}$ becomes

$$
\mathcal{H}: \quad \sin ^{2}\left(\frac{\theta}{2}\right)+\left(\frac{p}{2}\right)^{2}=\frac{e_{1}-e_{0}}{e_{0}-e_{3}} \cdot \frac{e_{2}-e_{3}}{e_{1}-e_{2}} \equiv m^{2} .
$$

$\mathcal{N}$ is interpreted as the cotangent space and has the geometry of a circular cylinder of unitary radius. On the other hand, according to equation (3.4), $\mathcal{H}$ is the Hamiltonian of a pendulum. This is an analog of the relation found by Holm and Marsden [35]. Since situation 1 in Table 2 is valid for any value of $e_{0}$ in the interval $e_{3}<e_{0}<e_{1}$, it follows that $0<m^{2}<1$ for $e_{0}>e_{2}$, $m^{2}=1$ for $e_{0}=e_{2}$ and $m^{2}>1$ for $e_{0}<e_{2}$ (see Table 1). Therefore, for $m^{2}<1$ we have an oscillatory movement of the pendulum, for $m^{2}=1$ we have a critical movement and for $m^{2}>1$ we have a circulating one. Figure $1 b$ shows the complete phase space of the pendulum.

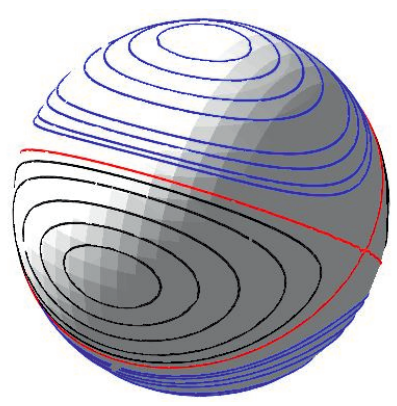

(a) Rigid body solutions

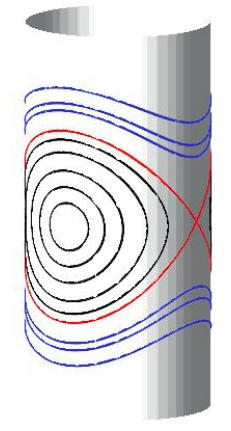

(b) Pendulum with Hamiltonian $\mathcal{H}$

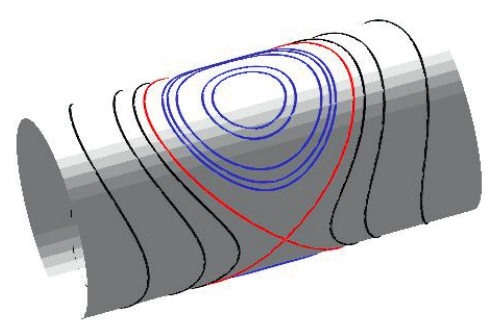

(c) Pendulum with Hamiltonian $\mathcal{N}$

Fig. 1. (a) The solution trajectories of the vector of angular momentum in the body-fixed frame, for the $\mathfrak{s o}(3)$ rigid body. The ones corresponding to $e_{2}<e_{0}$ are drawn in black, whereas the ones corresponding to $e_{0}<e_{2}$ are drawn in blue. The red lines correspond to the separatrixes. (b) The phase space of a simple pendulum with Hamiltonian $\mathcal{H}$ and cotangent space $\mathcal{N}$. The oscillatory movements are drawn in black, whereas the circulating ones are drawn in blue. The colors correspond to the ones in Fig. 1a and represent the relation between them. (c) The phase space of a simple pendulum with Hamiltonian $\mathcal{N}$ and cotangent space $\mathcal{H}$. The oscillatory trajectories are drawn in blue, whereas the circulating ones are drawn in black. The colors correspond to the ones in Fig. 1a and represent the relation between them.

Regarding the generators (2.32) of the system, it is straightforward to check that they can be redefined as

$$
\begin{aligned}
Y_{u_{1}} & \equiv \frac{1}{c \alpha} X_{u_{1}}=-\frac{\left(e_{1}-e_{3}\right)}{\alpha} k_{1}^{2} u_{2} \partial_{3}, \\
Y_{u_{2}} & \equiv \frac{k_{1}}{c \alpha} X_{u_{2}}=\frac{\left(e_{1}-e_{3}\right)}{\alpha} k_{1} u_{1} \partial_{3}, \\
Y_{u_{3}} & \equiv \frac{1}{c k_{1}\left(e_{1}-e_{3}\right)} X_{u_{3}}=k_{1} u_{2} \partial_{1}-\frac{1}{k_{1}} u_{1} \partial_{2},
\end{aligned}
$$

which satisfy the Lie-Poisson algebra iso(2)

$$
\left[Y_{u_{1}}, Y_{u_{2}}\right]=0, \quad\left[Y_{u_{2}}, Y_{u_{3}}\right]=Y_{u_{1}}, \quad\left[Y_{u_{3}}, Y_{u_{1}}\right]=Y_{u_{2}}
$$


Notice that in addition to the constant $c$ in (4.11), the generators $Y_{u_{1}}$ and $Y_{u_{2}}$ can be multiplied by an arbitrary constant $\alpha$.

A Lie-Poisson structure for the pendulum can be introduced on the cylindrical surface $\mathcal{N}=1[35]$, in terms of the variables $\theta, p$ by defining the Lie-Poisson bracket as

$$
\{f, g\}_{\mathcal{N}}=-\nabla \mathcal{N} \cdot(\nabla f \times \nabla g)
$$

A straightforward calculation gives

$$
\{f, g\}_{\mathcal{N}}=-8 \frac{\left(e_{1}-e_{3}\right)\left(e_{2}-e_{3}\right)}{\sqrt{\left(e_{1}-e_{2}\right)\left(e_{0}-e_{3}\right)}}\left(\frac{\partial f}{\partial \theta} \frac{\partial g}{\partial p}-\frac{\partial f}{\partial p} \frac{\partial g}{\partial \theta}\right)
$$

which shows that the variables $\theta$ and $p$ are canonically conjugate up to a scale factor: $\{p, \theta\}_{\mathcal{N}}=8 \frac{\left(e_{1}-e_{3}\right)\left(e_{2}-e_{3}\right)}{\sqrt{\left(e_{1}-e_{2}\right)\left(e_{0}-e_{3}\right)}}$. In terms of this bracket the canonical equations of motion are given by

$$
\frac{d \theta}{d \tau}=\{\mathcal{H}, \theta\}_{\mathcal{N}} \quad \text { and } \quad \frac{d p}{d \tau}=\{\mathcal{H}, p\}_{\mathcal{N}} .
$$

Combining these we obtain the Newton equation of motion for the simple pendulum

$$
\frac{d^{2} \theta}{d \tau^{2}}=-16 \frac{\left(e_{1}-e_{3}\right)^{2}\left(e_{2}-e_{3}\right)^{2}}{\left(e_{1}-e_{2}\right)\left(e_{0}-e_{3}\right)} \sin \theta
$$

- Simple pendulum with imaginary time and Hamiltonian $\mathcal{H}$ : Consider the momentum map

$$
u_{1} \equiv \sqrt{\frac{e_{0}-e_{3}}{e_{1}-e_{3}}} \sin \left(\frac{\theta^{\prime}}{2}\right), \quad u_{2} \equiv \frac{1}{k_{1}} \sqrt{\frac{e_{0}-e_{3}}{e_{1}-e_{3}}} \cos \left(\frac{\theta^{\prime}}{2}\right), \quad u_{3} \equiv i \frac{k_{2}}{k_{1}} \sqrt{\frac{e_{0}-e_{3}}{e_{1}-e_{3}}} \frac{\mathbb{P}}{2} .
$$

The expression for the level surface of the integral of motion $\mathcal{N}$ is again explicitly satisfied, whereas the form of the integral of motion $\mathcal{H}$ becomes

$$
\mathcal{H}: \quad \sin ^{2}\left(\frac{\theta^{\prime}}{2}\right)+\left(\frac{\mathbb{P}}{2}\right)^{2}=m_{c}^{2}
$$

where $m_{c}^{2}$ is the complementary modulus as defined in (2.18). The integral of motion $\mathcal{H}$ represents the Hamiltonian of the simple pendulum with imaginary time (3.18). Again because (4.18) is valid for any value of $e_{0}$, we have all different movements of the pendulum. In particular, for the oscillatory motions: $e_{2}<e_{0}$ and $0<m_{c}^{2}<1$, for the asymptotical motion: $e_{0}=e_{2}$ and $m_{c}^{2}=0$, whereas for the circulating movements: $e_{0}<e_{2}$ and $m_{c}^{2}<0$ (see Table 1 ). Notice that the explicit presence of the imaginary number $i$ in coordinate $u_{3}$ geometrically plays the role of changing the elliptic cylinder into a hyperbolic cylinder, although the former is defined in a real space $\mathbb{R}^{3}$, whereas the latter is defined in a complex space $\mathbb{R}^{2} \times i \mathbb{R}$. Regarding the Lie-Poisson algebra, it is clear that here we have the same iso(2) algebra (4.12). The two-dimensional Lie-Poisson bracket in this case has a purely imaginary scale factor

$$
\{f, g\}_{\mathcal{N}}=-8 i \frac{\left(e_{1}-e_{3}\right)\left(e_{2}-e_{3}\right)}{\sqrt{\left(e_{1}-e_{2}\right)\left(e_{0}-e_{3}\right)}}\left(\frac{\partial f}{\partial \theta} \frac{\partial g}{\partial \mathbb{P}}-\frac{\partial f}{\partial \mathbb{P}} \frac{\partial g}{\partial \theta}\right)
$$


which is in agreement with the fact that the Newton equation for the simple pendulum with imaginary time has an extra minus sign in the force term

$$
\frac{d^{2} \theta}{d \tau^{2}}=16 \frac{\left(e_{1}-e_{3}\right)^{2}\left(e_{2}-e_{3}\right)^{2}}{\left(e_{1}-e_{2}\right)\left(e_{0}-e_{3}\right)} \sin \theta
$$

Because the rigid body is a bi-Hamiltonian system, it is interesting to obtain the simple pendulum, but now having the integral of motion $\mathcal{N}$ as the Hamiltonian and the integral of motion $\mathcal{H}$ as the cotangent space.

- Simple pendulum with real time and Hamiltonian $\mathcal{N}$ : Consider the momentum map

$$
u_{1} \equiv \frac{k_{1}}{k_{2}} \sqrt{\frac{e_{1}-e_{0}}{e_{1}-e_{3}}} \frac{p}{2}, \quad u_{2} \equiv \frac{1}{k_{2}} \sqrt{\frac{e_{1}-e_{0}}{e_{1}-e_{3}}} \sin \left(\frac{\theta}{2}\right), \quad u_{3} \equiv \sqrt{\frac{e_{1}-e_{0}}{e_{1}-e_{3}}} \cos \left(\frac{\theta}{2}\right) .
$$

Now the expression for the integral of motion $\mathcal{H}$ is explicitly satisfied, whereas the expression for the integral of motion $\mathcal{N}$ becomes

$$
\mathcal{N}: \quad\left(\frac{p}{2}\right)^{2}+\sin ^{2}\left(\frac{\theta}{2}\right)=\frac{1}{m^{2}}
$$

which, according to Eq. (3.4), corresponds to the energy of a simple pendulum. Because situation 8 in Table 3 is valid for any value of $e_{0}$ in the interval $e_{3}<e_{0}<e_{1}$, in equation (4.22) we have all different movements of the pendulum. It is worth stressing that, whereas for values $e_{0}<e_{2}$ for which $0<m^{2}<1$ the movements in (4.10) are oscillatory (see Fig. 1b), in (4.22) for the same values of $e_{0}: 1<1 / m^{2}<\infty$ and therefore we have circulating pendulums (see Fig. 1c). Something similar occurs for the circulating movements in (4.10) and the oscillatory ones in (4.22). The asymptotical cases are given for $e_{0}=e_{2}$ for which $m^{2}=1$ in both (4.10) and (4.22).

Let us emphasize that the circular cylinder $\mathcal{H}$ has unitary radius. In this case the Hamiltonian vector fields associated with the coordinates $u_{i}$ 's are given by Eqs. (2.35). Redefining these vector fields as

$$
\begin{aligned}
\widetilde{Y}_{u_{1}} & \equiv \frac{1}{a\left(e_{1}-e_{3}\right) k_{2}} \widetilde{X}_{u_{1}}=\frac{1}{k_{2}} u_{3} \partial_{2}-k_{2} u_{2} \partial_{3}, \\
\widetilde{Y}_{u_{2}} & \equiv \frac{1}{a \alpha} \widetilde{X}_{u_{2}}=-\frac{\left(e_{1}-e_{3}\right)}{\alpha} u_{3} \partial_{1}, \\
\widetilde{Y}_{u_{3}} & \equiv \frac{1}{a \alpha k_{2}} \widetilde{X}_{u_{3}}=\frac{e_{1}-e_{3}}{\alpha} k_{2} u_{2} \partial_{1},
\end{aligned}
$$

they satisfy the $\mathfrak{i s o}(2)$ Lie-Poisson algebra

$$
\left[\widetilde{Y}_{u_{1}}, \widetilde{Y}_{u_{2}}\right]=\widetilde{Y}_{u_{3}}, \quad\left[\widetilde{Y}_{u_{2}}, \widetilde{Y}_{u_{3}}\right]=0, \quad\left[\tilde{Y}_{u_{3}}, \widetilde{Y}_{u_{1}}\right]=\widetilde{Y}_{u_{2}}
$$

In $(4.23) \alpha$ is an arbitrary constant.

Regarding the Lie-Poisson bracket in terms of the variables $(\theta, p)$ on the cylindrical surface $\mathcal{H}=1$, and defined as

$$
\{f, g\}_{\mathcal{H}}=-\nabla \mathcal{H} \cdot(\nabla f \times \nabla g)
$$

we have

$$
\{f, g\}_{\mathcal{H}}=8 \frac{\left(e_{1}-e_{3}\right)\left(e_{1}-e_{2}\right)}{\sqrt{\left(e_{1}-e_{0}\right)\left(e_{2}-e_{3}\right)}}\left(\frac{\partial f}{\partial \theta} \frac{\partial g}{\partial p}-\frac{\partial f}{\partial p} \frac{\partial g}{\partial \theta}\right) .
$$


The Newton equation associated to this Lie-Poisson bracket is

$$
\frac{d^{2} \theta}{d \tau^{2}}=-16 \frac{\left(e_{1}-e_{3}\right)^{2}\left(e_{1}-e_{2}\right)^{2}}{\left(e_{1}-e_{0}\right)\left(e_{2}-e_{3}\right)} \sin \theta
$$

- Simple pendulum with imaginary time and Hamiltonian $\mathcal{N}$ : Consider the momentum map

$$
u_{1} \equiv i \frac{k_{1}}{k_{2}} \sqrt{\frac{e_{1}-e_{0}}{e_{1}-e_{3}}} \frac{\mathbb{P}}{2}, \quad u_{2} \equiv \frac{1}{k_{2}} \sqrt{\frac{e_{1}-e_{0}}{e_{1}-e_{3}}} \cos \left(\frac{\theta^{\prime}}{2}\right), \quad u_{3} \equiv \sqrt{\frac{e_{1}-e_{0}}{e_{1}-e_{3}}} \sin \left(\frac{\theta^{\prime}}{2}\right) .
$$

The expression for the integral of motion $\mathcal{H}$ is explicitly satisfied, whereas the expression for the integral of motion $\mathcal{N}$ becomes

$$
\mathcal{N}: \quad\left(\frac{\mathbb{P}}{2}\right)^{2}+\sin ^{2}\left(\frac{\theta^{\prime}}{2}\right)=-\frac{m_{c}^{2}}{m^{2}} .
$$

This is the Hamiltonian of a simple pendulum with imaginary time. As in the previous cases, because (4.29) is valid for any value of $e_{0}$, it includes all different movements of the pendulum. Because the cotangent space is $\mathcal{H}$, the $\mathfrak{i s o}(2)$ Lie-Poisson algebra is given by (4.24), and the LiePoisson bracket in terms of the variables $(\theta, \mathbb{P})$ is similar to $(4.26)$ and differs only by a factor of $i$ in the scale factor.

The geometrical interpretation of these results is straightforward. If we start with a specific $\mathfrak{s o}(3)$ rigid body, the three moments of inertia $\left\{I_{i}\right\}$ are given, which fix the parameter $\kappa$ and therefore the three dimensionless parameters of inertia $\left\{e_{i}\right\}$. The different solutions are obtained for different values of $e_{0}$. After the $S L(2, \mathbb{R})$ gauge transformation of the level surfaces of the integrals of motion, for the momentum maps (4.9) and (4.17) we obtain a unitary circular cylinder $\mathcal{N}$ with the axis along the $u_{3}$ direction representing the cotangent space. On the other hand, geometrically $\mathcal{H}$ is an element of a set of elliptic-(hyperbolic) cylinders with axis along the $u_{1}$ direction which physically are energy surfaces, because $\mathcal{H}$ is the Hamiltonian of the simple pendulum (see Eqs. (3.4) and (3.18)). Intersections of the single circular cylinder $\mathcal{N}$ with the set of elliptic-(hyperbolic) cylinders $\mathcal{H}$ represent all the movements of the pendulum. On the other hand, for the momentum maps (4.21) and (4.28) the surface $\mathcal{H}$ becomes an unitary circular cylinder with axis along the $u_{1}$ direction representing the cotangent space and $\mathcal{N}$ is an element of a set of elliptic-(hyperbolic) cylinders that represent the Hamiltonian of the simple pendulum.

\subsection{Intersection of a hyperbolic cylinder and an elliptic cylinder I}

Let us consider the cases 2-8 and 3-8, which correspond to the intersection of a hyperbolic cylinder and an elliptical cylinder. In these cases the $S L(2, \mathbb{R})$ group element has the form

$$
g=\left(\begin{array}{cc}
\frac{1}{c\left(e_{2}-e_{3}\right)} & -\frac{e_{2}}{c\left(e_{2}-e_{3}\right)} \\
c & -c e_{2}
\end{array}\right) .
$$

As a consequence, the level surfaces of the integrals of motion are given by the expressions

$$
\begin{array}{ll}
\mathcal{H}: & k_{2}^{2} u_{1}^{2}-k_{1}^{2} u_{3}^{2}=\frac{e_{0}-e_{2}}{e_{1}-e_{3}}, \\
\mathcal{N}: & u_{1}^{2}+k_{1}^{2} u_{2}^{2}=\frac{e_{0}-e_{3}}{e_{1}-e_{3}} .
\end{array}
$$




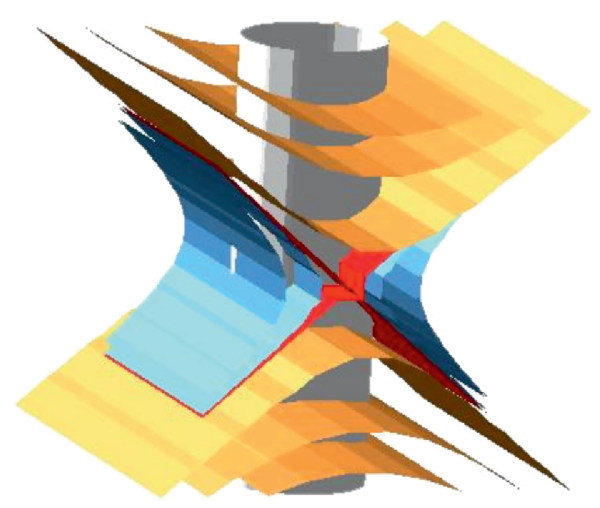

Fig. 2. The figure shows the geometry of the hyperbolic cylinders for different values of $e_{0}$. The geometry corresponding to $e_{2}<e_{0}$ is drawn in blue, whereas the ones corresponding to $e_{0}<e_{2}$ are drawn in yellow. The red planes correspond to the separatrixes.

Notice that the integral of motion $\mathcal{N}$ is exactly the same as (4.8), as it should be since in both cases we are dealing with situation 8 (see Table 3), which represents an elliptic cylinder of unitary radius with axis along $u_{3}$. Cases 2-8 and 3-8 differ in the sign of the right-hand side of the integral of motion $\mathcal{H}(4.31)$, for case $2-8$ we have $e_{0}-e_{2}>0$, whereas for case $3-8$ we have $e_{0}-e_{2}<0$. Geometrically the different signs change the orientation of the hyperbolic cylinders (see Fig. 2). Again we expect to have two different momentum maps, one in which $\mathcal{N}$ is the cotangent space and $\mathcal{H}$ is the Hamiltonian and the other where $\mathcal{H}$ is the cotangent space and $\mathcal{N}$ is the Hamiltonian.

- Simple pendulum with real time and Hamiltonian $\mathcal{H}$ : As we have discussed previously, under the momentum map (4.9)

$$
u_{1} \equiv \sqrt{\frac{e_{0}-e_{3}}{e_{1}-e_{3}}} \cos \left(\frac{\theta}{2}\right), \quad u_{2} \equiv \frac{1}{k_{1}} \sqrt{\frac{e_{0}-e_{3}}{e_{1}-e_{3}}} \sin \left(\frac{\theta}{2}\right), \quad u_{3} \equiv \frac{k_{2}}{k_{1}} \sqrt{\frac{e_{0}-e_{3}}{e_{1}-e_{3}}}\left(\frac{p}{2}\right),
$$

the expression for the integral of motion $\mathcal{N}$ is automatically satisfied. On the other hand, the level surface of the integral of motion (4.31) coincides with the Hamiltonian (3.4) and (4.10)

$$
\mathcal{H}: \quad \sin ^{2}\left(\frac{\theta}{2}\right)+\left(\frac{p}{2}\right)^{2}=m^{2},
$$

where $0<m^{2}<1$ for case 2-8 $\left(e_{0}-e_{2}>0\right)$ and therefore represents the oscillatory movements, whereas $1<m^{2}<\infty$ for case $3-8\left(e_{0}-e_{2}<0\right)$ which represents the circulating movements. As usual, the case $e_{0}=e_{2}$ produces the separatrix. For the Hamiltonian vector fields associated to the coordinates, they are the same as (4.11) and therefore they satisfy the Lie-Poisson algebra $\mathfrak{i s o}(2)$ (4.12). A straightforward calculation gives the two-dimensional Lie-Poisson bracket (4.14) and the Newton equation (4.16). These results show that this case and IV-A represent the same dynamics, although they come from a different geometry for the integral of motion $\mathcal{H}$.

- Simple pendulum with imaginary time and Hamiltonian $\mathcal{H}$ : As in Section 4.1, under the momentum map

$$
u_{1} \equiv \sqrt{\frac{e_{0}-e_{3}}{e_{1}-e_{3}}} \sin \left(\frac{\theta^{\prime}}{2}\right), \quad u_{2} \equiv \frac{1}{k_{1}} \sqrt{\frac{e_{0}-e_{3}}{e_{1}-e_{3}}} \cos \left(\frac{\theta^{\prime}}{2}\right), \quad u_{3} \equiv i \frac{k_{2}}{k_{1}} \sqrt{\frac{e_{0}-e_{3}}{e_{1}-e_{3}}}\left(\frac{\mathbb{P}}{2}\right)
$$


the expression for the integral of motion $\mathcal{N}$ is automatically satisfied, whereas the integral of motion $\mathcal{H}$ becomes

$$
\mathcal{H}: \quad \sin ^{2}\left(\frac{\theta^{\prime}}{2}\right)+\left(\frac{\mathbb{P}}{2}\right)^{2}=m_{c}^{2},
$$

where $-\infty<m_{c}^{2}<1$. Notice that the geometrical role of writing the coordinate $u_{3}$ in terms of an imaginary momentum $\mathbb{P}$ is to map the hyperbolic cylinder $\mathcal{H}$ to an elliptic cylinder with axis along the $u_{2}$ direction. According to Eq. (3.18), $\mathcal{H}$ represents the Hamiltonian for a simple pendulum of imaginary time and energy $m^{2}=1-m_{c}^{2}$, as the one in (4.2). As expected, the twodimensional Lie-Poisson structure and the Newton equations coincide with (4.19) and (4.20).

The next natural mapping is the one that maps the rigid body system to a cotangent space $\mathcal{H}$, with Hamiltonian $\mathcal{N}$. By inspection of the integral of motion (4.31), this can be achieved by introducing a purely imaginary coordinate. Doing this allows the hyperbolic nature of the cylinders defined in $\mathbb{R}^{3}$ to be changed to elliptic cylinders defined in $\mathbb{R}^{2} \times i \mathbb{R}$.

- Circulating simple pendulum with real time, imaginary coordinate and Hamiltonian $\mathcal{N}$ : Consider the momentum map

$$
u_{1} \equiv \frac{1}{k_{2}} \sqrt{\frac{e_{0}-e_{2}}{e_{1}-e_{3}}} \sin \left(\frac{\theta}{2}\right), \quad u_{2} \equiv \frac{1}{k_{1} k_{2}} \sqrt{\frac{e_{0}-e_{2}}{e_{1}-e_{3}}} \frac{p}{2}, \quad u_{3} \equiv \frac{i}{k_{1}} \sqrt{\frac{e_{0}-e_{2}}{e_{1}-e_{3}}} \cos \left(\frac{\theta}{2}\right) .
$$

Here we assume: $e_{0}-e_{2}>0$ (situation 2 in Table 2). For such a map the integral of motion $\mathcal{H}$ is automatically satisfied, whereas the integral of motion $\mathcal{N}$ takes the form

$$
\mathcal{N}: \quad \sin ^{2}\left(\frac{\theta}{2}\right)+\left(\frac{p}{2}\right)^{2}=\frac{1}{m_{c}^{2}}
$$

where $1<1 / m_{c}^{2}<\infty$ (see Table 1 ) and therefore the Hamiltonian equation represents only circulating movements of the simple pendulum.

Because the Hamiltonian is given by $\mathcal{N}$, the suitable Hamiltonian vector fields associated to the coordinates are given by Eq. (2.35). Redefining them as

$$
\begin{aligned}
\widetilde{Y}_{u_{1}} & \equiv \frac{k_{2}}{a \beta k_{1}} \widetilde{X}_{u_{1}}=\frac{1}{\beta} \sqrt{\left(e_{1}-e_{2}\right)\left(e_{2}-e_{3}\right)} u_{3} \partial_{2}, \\
\widetilde{Y}_{u_{2}} & \equiv \frac{1}{a \sqrt{\left(e_{1}-e_{3}\right)\left(e_{2}-e_{3}\right)}} \widetilde{X}_{u_{2}}=-\frac{k_{2}}{k_{1}} u_{1} \partial_{2}-\frac{k_{1}}{k_{2}} u_{3} \partial_{1}, \\
\widetilde{Y}_{u_{3}} & \equiv \frac{1}{a \beta} \widetilde{X}_{u_{3}}=\frac{1}{\beta}\left(e_{1}-e_{2}\right) u_{1} \partial_{2},
\end{aligned}
$$

they satisfy the $\mathfrak{i s o}(1,1)$ Lie-Poisson algebra

$$
\left[\widetilde{Y}_{u_{1}}, \widetilde{Y}_{u_{2}}\right]=\widetilde{Y}_{u_{3}}, \quad\left[\widetilde{Y}_{u_{2}}, \widetilde{Y}_{u_{3}}\right]=-\widetilde{Y}_{u_{1}}, \quad\left[\tilde{Y}_{u_{3}}, \widetilde{Y}_{u_{1}}\right]=0 .
$$

Notice that, in addition to the constant $a$ in (4.37), the generators $\widetilde{Y}_{u_{1}}$ and $\widetilde{Y}_{u_{3}}$ can be multiplied by an arbitrary constant $\beta$. For the two-dimensional Lie-Poisson bracket, it has the form

$$
\{f, g\}_{\mathcal{N}}=-8 i \frac{\left(e_{1}-e_{2}\right)\left(e_{2}-e_{3}\right)}{\sqrt{\left(e_{0}-e_{2}\right)\left(e_{1}-e_{3}\right)}}\left(\frac{\partial f}{\partial \theta} \frac{\partial g}{\partial p}-\frac{\partial f}{\partial p} \frac{\partial g}{\partial \theta}\right)
$$


while the equation of motion is

$$
\frac{d^{2} \theta}{d \tau^{2}}=16 \frac{\left(e_{2}-e_{3}\right)^{2}\left(e_{1}-e_{2}\right)^{2}}{\left(e_{1}-e_{3}\right)\left(e_{0}-e_{2}\right)} \sin \theta,
$$

At this point the sign in the Newton equation seems to be wrong, but this sign reflects the fact that we have applied a complex map in coordinate $u_{3}$.

Although we have presented the analysis assuming $e_{2}-e_{0}<0$, it is worth pointing out that the same physical situation arises also for the case $e_{2}-e_{0}>0$. The only difference is to introduce a mapping where the complex coordinate is $u_{1}$ instead of $u_{3}$. As a conclusion of this subsection, we find that, for the situation where the intersection of integrals of motion is between a hyperbolic cylinder $\mathcal{H}$ and an elliptic cylinder $\mathcal{N}$, if the Hamiltonian is given by $\mathcal{H}$ and the cotangent space is given by $\mathcal{N}$, then we get the whole motions of the simple pendulum and the Lie algebra of the extended rigid body is iso(2), but in the case where $\mathcal{N}$ is the Hamiltonian and $\mathcal{H}$ is the cotangent space we only get the circulating motions and the Lie algebra is iso $(1,1)$.

- Circulating simple pendulum with imaginary time, imaginary coordinate and Hamiltonian $\mathcal{N}$ : Consider the mapping

$$
u_{1} \equiv \frac{1}{k_{2}} \sqrt{\frac{e_{0}-e_{2}}{e_{1}-e_{3}}} \cos \left(\frac{\theta^{\prime}}{2}\right), \quad u_{2} \equiv \frac{i}{k_{1} k_{2}} \sqrt{\frac{e_{0}-e_{2}}{e_{1}-e_{3}}} \frac{\mathbb{P}}{2}, \quad u_{3} \equiv \frac{i}{k_{1}} \sqrt{\frac{e_{0}-e_{2}}{e_{1}-e_{3}}} \sin \left(\frac{\theta^{\prime}}{2}\right)
$$

For such a map, where we are assuming $e_{0}-e_{2}>0$, the integral of motion $\mathcal{H}$ is automatically satisfied, whereas the integral of motion $\mathcal{N}$ takes the form

$$
\mathcal{N}: \quad\left(\frac{\mathbb{P}}{2}\right)^{2}+\sin ^{2}\left(\frac{\theta^{\prime}}{2}\right)=-\frac{m^{2}}{m_{c}^{2}}
$$

It is straightforward to notice that $-m^{2} / m_{c}^{2}<0$, and therefore we have, as expected, only circulating movements. This case also comes from the extended rigid body with Lie algebra iso $(1,1)$. For the two-dimensional Lie-Poisson bracket it has the following expression:

$$
\{f, g\}_{\mathcal{N}}=8 \frac{\left(e_{1}-e_{2}\right)\left(e_{2}-e_{3}\right)}{\sqrt{\left(e_{0}-e_{2}\right)\left(e_{1}-e_{3}\right)}}\left(\frac{\partial f}{\partial \theta} \frac{\partial g}{\partial \mathbb{P}}-\frac{\partial f}{\partial \mathbb{P}} \frac{\partial g}{\partial \theta}\right)
$$

while the Newton equation of motion goes as (4.40), but with an extra minus sign on the righthand side of the equation. Again the discrepancy between the Newton equation and Eq. (3.18) is understood by the fact that the momentum map (4.41) considers a complex transformation in coordinate $u_{3}$.

\subsection{Intersection of a hyperbolic cylinder and an elliptic cylinder II}

As final cases we study the intersections between the elliptic cylinder and hyperbolic cylinder 1-6 and 1-7, for which the $S L(2, \mathbb{R})$ group elements take the form

$$
g=\left(\begin{array}{cc}
\frac{1}{c\left(e_{1}-e_{3}\right)} & -\frac{e_{1}}{c\left(e_{1}-e_{3}\right)} \\
c & -c e_{2}
\end{array}\right) .
$$


For these intersections the expressions for the integrals of motion are

$$
\begin{array}{ll}
\mathcal{H}: & k_{2}^{2} u_{2}^{2}+u_{3}^{2}=\frac{e_{1}-e_{0}}{e_{1}-e_{3}}, \\
\mathcal{N}: & -k_{2}^{2} u_{1}^{2}+k_{1}^{2} u_{3}^{2}=\frac{e_{2}-e_{0}}{e_{1}-e_{3}} .
\end{array}
$$

The surface $\mathcal{H}$ has the same expression as (4.7) as it should be since we are working with situation 1 of Table 2, which corresponds to an elliptic cylinder.

- Simple pendulum with real time and Hamiltonian $\mathcal{N}$ : Consider the momentum map (4.21)

$$
u_{1} \equiv \frac{k_{1}}{k_{2}} \sqrt{\frac{e_{1}-e_{0}}{e_{1}-e_{3}}} \frac{p}{2}, \quad u_{2} \equiv \frac{1}{k_{2}} \sqrt{\frac{e_{1}-e_{0}}{e_{1}-e_{3}}} \sin \left(\frac{\theta}{2}\right), \quad u_{3} \equiv \sqrt{\frac{e_{1}-e_{0}}{e_{1}-e_{3}}} \cos \left(\frac{\theta}{2}\right) .
$$

Clearly, the integral of motion $\mathcal{H}$ is automatically satisfied, whereas the integral of motion is

$$
\mathcal{N}: \quad\left(\frac{p}{2}\right)^{2}+\sin ^{2}\left(\frac{\theta}{2}\right)=\frac{1}{m^{2}} .
$$

For values of $e_{0}<e_{2}$, the quotient $1 / m^{2}<1$ and therefore $\mathcal{N}$ represents the Hamiltonian for the simple pendulum in oscillatory motion, whereas for $e_{0}>e_{2}, 1 / m^{2}>1$ and $\mathcal{N}$ represents the Hamiltonian for the simple pendulum in circulating motion (3.4). For the limiting case $e_{0}=e_{2}$, $1 / m^{2}=1$ and we obtain the asymptotic motion.

As expected, in this case the two-dimensional Lie-Poisson bracket coincides with (4.26) and therefore the Newton equation of motion is given by (4.27).

- Simple pendulum with imaginary time and Hamiltonian $\mathcal{N}$ : Consider the momentum $\operatorname{map}(4.28)$

$$
u_{1} \equiv i \frac{k_{1}}{k_{2}} \sqrt{\frac{e_{1}-e_{0}}{e_{1}-e_{3}}} \frac{\mathbb{P}}{2}, \quad u_{2} \equiv \frac{1}{k_{2}} \sqrt{\frac{e_{1}-e_{0}}{e_{1}-e_{3}}} \cos \left(\frac{\theta^{\prime}}{2}\right), \quad u_{3} \equiv \sqrt{\frac{e_{1}-e_{0}}{e_{1}-e_{3}}} \sin \left(\frac{\theta^{\prime}}{2}\right) .
$$

Under this map the integral of motion $\mathcal{H}$ is automatically satisfied, whereas the integral of motion $\mathcal{N}$ becomes

$$
\mathcal{N}: \quad\left(\frac{\mathbb{P}}{2}\right)^{2}+\sin ^{2}\left(\frac{\theta^{\prime}}{2}\right)=-\frac{m_{c}^{2}}{m^{2}},
$$

which is precisely the Hamiltonian of simple pendulum with imaginary time (3.18). Because we are using the same cotangent space (4.7) and (4.45), the rest of physical quantities such as the two-dimensional Lie-Poisson bracket and the Newton equation of motion coincide with those mentioned below Eq. (4.29).

Following the previous cases, the next natural situation is the one that maps the rigid body system to a cotangent space $\mathcal{N}$, with Hamiltonian $\mathcal{H}$. By inspection of the integral of motion (4.46), this can be achieved by introducing a momentum map with a purely imaginary coordinate.

- Circulating pendulum with real time, imaginary coordinate and Hamiltonian $\mathcal{H}$ : Consider the momentum map

$$
u_{1} \equiv \frac{i}{k_{2}} \sqrt{\frac{e_{2}-e_{0}}{e_{1}-e_{3}}} \cos \left(\frac{\theta}{2}\right), \quad u_{2} \equiv \frac{1}{k_{1} k_{2}} \sqrt{\frac{e_{2}-e_{0}}{e_{1}-e_{3}}} \frac{p}{2}, \quad u_{3} \equiv \frac{1}{k_{1}} \sqrt{\frac{e_{2}-e_{0}}{e_{1}-e_{3}}} \sin \left(\frac{\theta}{2}\right) .
$$

Here we are assuming $e_{2}-e_{0}>0$ (situation 7 in Table 3 ). 
Under this map the integral of motion $\mathcal{N}$ is satisfied, while the Hamiltonian $\mathcal{H}$ has the following expression:

$$
\mathcal{H}: \quad\left(\frac{p}{2}\right)^{2}+\sin ^{2}\left(\frac{\theta}{2}\right)=-\frac{m^{2}}{m_{c}^{2}} .
$$

For values $e_{2}-e_{0}>0$ the quotient $1<-m^{2} / m_{c}^{2}<\infty$ (see Table 1 ) and we have a simple pendulum in circulating motion. For the two-dimensional Lie-Poisson bracket it has the following expression:

$$
\{f, g\}_{\mathcal{N}}=8 i \frac{\left(e_{1}-e_{2}\right)\left(e_{2}-e_{3}\right)}{\sqrt{\left(e_{2}-e_{0}\right)\left(e_{1}-e_{3}\right)}}\left(\frac{\partial f}{\partial \theta} \frac{\partial g}{\partial p}-\frac{\partial f}{\partial p} \frac{\partial g}{\partial \theta}\right)
$$

whereas the Newton equation of motion reads

$$
\frac{d^{2} \theta}{d \tau^{2}}=16 \frac{\left(e_{1}-e_{2}\right)^{2}\left(e_{2}-e_{3}\right)^{2}}{\left(e_{2}-e_{0}\right)\left(e_{1}-e_{3}\right)} \sin \theta
$$

As in the analogous physical situation in Section 4.2, the extra minus sign that appears in the Newton equation (4.53) is due to the complex nature of the map (4.50). As for the case $e_{0}>e_{2}$, we can go through it, but the physical result will be the same, we only get the circulating movements of the pendulum.

- Circulating pendulum with imaginary time, imaginary coordinate and Hamiltonian $\mathcal{H}$ : Consider the momentum map in which we assume $e_{2}>e_{0}$

$$
u_{1} \equiv \frac{i}{k_{2}} \sqrt{\frac{e_{2}-e_{0}}{e_{1}-e_{3}}} \sin \left(\frac{\theta^{\prime}}{2}\right), \quad u_{2} \equiv \frac{i}{k_{1} k_{2}} \sqrt{\frac{e_{2}-e_{0}}{e_{1}-e_{3}}} \frac{\mathbb{P}}{2}, \quad u_{3} \equiv \frac{1}{k_{1}} \sqrt{\frac{e_{2}-e_{0}}{e_{1}-e_{3}}} \cos \left(\frac{\theta^{\prime}}{2}\right)
$$

Under this map the integral of motion $\mathcal{N}$ is satisfied, while the Hamiltonian $\mathcal{H}$ has the following expression:

$$
\mathcal{H}: \quad\left(\frac{\mathbb{P}}{2}\right)^{2}+\sin ^{2}\left(\frac{\theta^{\prime}}{2}\right)=\frac{1}{m_{c}^{2}} .
$$

It is clear that $-\infty<1 / m_{c}^{2}<0$ and therefore, as expected, we only have circulating motions.

For completeness, the two-dimensional Lie-Poisson bracket is

$$
\{f, g\}_{\mathcal{N}}=-8 \frac{\left(e_{1}-e_{2}\right)\left(e_{2}-e_{3}\right)}{\sqrt{\left(e_{2}-e_{0}\right)\left(e_{1}-e_{3}\right)}}\left(\frac{\partial f}{\partial \theta} \frac{\partial g}{\partial \mathbb{P}}-\frac{\partial f}{\partial \mathbb{P}} \frac{\partial g}{\partial \theta}\right)
$$

whereas the equation of motion reads

$$
\frac{d^{2} \theta}{d \tau^{2}}=-16 \frac{\left(e_{1}-e_{2}\right)^{2}\left(e_{2}-e_{3}\right)^{2}}{\left(e_{2}-e_{0}\right)\left(e_{1}-e_{3}\right)} \sin \theta
$$

Notice again the extra minus sign in (4.57) due to the complex nature of the map (4.54).

\section{The pendulum from the extended rigid body $(c \neq 0$ and $d=0)$}

Finally, let us analyze the second general set of $S L(2, \mathbb{R})$ transformations. Regarding the conditions (4.1) the second one becomes modified to $c e_{i}=0$. Since in this case $c \neq 0$, we have necessarily $e_{i}=0$. Even more, because we are restricted to the interval $\kappa \in(0, \pi / 3)$ the only possibility is to have $e_{2}=0$ and because of the restrictions (2.9) we also have $e_{1}=-e_{3}=\sqrt{3} / 2$. 
We conclude that conditions (4.1) can be satisfied only in two situations. In both of them $e_{2}=0$ and either $a e_{1}+b=0$ or $a e_{3}+b=0$. Due to the relation between $e_{1}$ and $e_{3}$ these two situations correspond to the transformation (2.27) with different signs of the coefficient $a$. Thus, without loss of generality we can restrict ourselves to the case $a>0$ and analyze one of the situations. The second one can be obtained from the same formulas by changing the sign of $a$. In summary, conditions (2.9) become

$$
a e_{1}+b=0, \quad e_{2}=0,
$$

and a generic $S L(2, \mathbb{R})$ element has the form

$$
g=\left(\begin{array}{cc}
\frac{1}{c e_{1}} & -\frac{1}{c} \\
c & 0
\end{array}\right)
$$

Notice that this transformation can be obtained from (4.44) modulo a factor of $1 / 2$ in the first arrow of the matrix, which does not change the geometry of the level surface of the integral of motion $\mathcal{H}$ and therefore (4.44) corresponds to the $S L(2, \mathbb{R})$ transformation that takes the original level surfaces of the integrals of motion to an elliptic and a hyperbolic cylinder. Notice that, for $e_{1}=-e_{3}$ and $e_{2}=0$, the quotients (2.19) become equal to $k_{1}^{2}=k_{2}^{2}=1 / 2$.

It is clear that we do not have to develop this case further, since we can obtain it from a limiting case of those studied previously. Here we have two situations again, either $e_{0}<0$ (situation 1-7 of Section 4.3) or $e_{0}>0$ (situation 1-6 of Section 4.3).

\section{Conclusions}

In this paper we have revisited the relation between the extended rigid body and the simple pendulum with the aim to give an exhaustive list of all different ways in which the relation takes place, both in real and imaginary time. We started in Section 2 reviewing the basics of the rigid body system in its two parameters formulation. The first parameter $e_{0}$ is related to the quotient $E / L^{2}$, where $E$ is the energy of the motion and $L^{2}$ is the square of the magnitude of angular momentum. The second parameter $\kappa$ codifies the values of the three moments of inertia. We work in this formulation of the rigid body because it allows us to have a good control over the different geometries of the two integrals of motion of the system [39].

The original construction to establish the relation between the extended rigid body and the pendulum was discussed by Holm and Marsden [35] and uses the $S L(2, \mathbb{R})$ symmetry of the Euler equations to find linear combinations of the two integrals of motion and transform them to new ones, denoted in this paper as $\mathcal{H}$ and $\mathcal{N}$. For one specific class of $S L(2, \mathbb{R})$ transformations, both integrals of motion have the geometry of an elliptic cylinder, this case corresponds to the extended rigid body with iso(2) Lie algebra. By a proper change of coordinates, and taking one of the cylinders as the cotangent space and the other as the Hamiltonian, it is possible to obtain both the two-dimensional Hamiltonian of the simple pendulum and its canonical equations of motion, even more, taking different values for the principal moments of inertia allows one to get the different solutions of the simple pendulum: oscillatory, circulating and critical. Our present work revisits the construction proposed by Holm and Marsden in the two parameters formulation and, since we are working with a bi-Hamiltonian system, we give the construction for the two different physical situations, i.e., when $\mathcal{N}$ is the cotangent space and $\mathcal{H}$ is the Hamiltonian and the situation where we invert the role of the integrals of motion, $\mathcal{H}$ as the cotangent space and $\mathcal{N}$ as the Hamiltonian. This exercise allows us to understand in a precise 
way what kind of movements in the rigid body are mapped to a certain type of movements of the simple pendulum. Specifically, we show that movements in the rigid body for which $e_{2}<e_{0}$ are mapped to oscillatory movements of the pendulum if $\mathcal{H}$ is the Hamiltonian, but are mapped to circulating movements of the pendulum if $\mathcal{N}$ is the Hamiltonian instead. Something similar occurs for movements with $e_{2}>e_{0}$.

Going one step beyond and using the whole $S L(2, \mathbb{R})$ transformations of the Euler equations, in this paper we study all different cases in which the solutions of the extended rigid body and those of the simple pendulum are related. We must emphasize that, when working out the reduction of the phase space, we take into account both time and spatial change of coordinates, which has allowed us to gain a better insight into the kind of dynamics the pendulums have, given the geometry. We have found that, if we keep the geometry of one of the integrals of motion in the form of an elliptic cylinder and consider the other integral of motion to be a hyperbolic cylinder, it is also possible to get the simple pendulum. To be specific, if $\mathcal{H}$ represents the elliptic cylinder and $\mathcal{N}$ represents the hyperbolic cylinder, then we have again two situations: i) If $\mathcal{H}$ is the cotangent space and $\mathcal{N}$ is the Hamiltonian, we can provide a change of coordinates that gives origin to the whole movements of the pendulum; this case also corresponds to an extended rigid body with Lie algebra iso(2). ii) If instead $\mathcal{H}$ is the Hamiltonian and $\mathcal{N}$ is the cotangent space, then we can provide a change of coordinates considering one of the coordinates as purely imaginary in such a way that we get the Hamiltonian of the pendulum, although the energies of the system correspond only to the circulating movements and not to the oscillatory ones; this case corresponds to the extended rigid body with Lie algebra iso $(1,1)$, the same situation occurs when we take two of the momenta as imaginary. To the best of our knowledge this is a physical situation not previously discussed in the literature. In every case we also give the reduction that relates the extended rigid body to the simple pendulum with imaginary time.

As for future work, because the analysis performed in this paper has been established only classically it would be interesting to extend the construction to the quantum framework. We expect that this analysis could produce interesting relations between the Lame differential equation, which governs the quantum behavior of the rigid body, and the Mathieu differential equation, which governs the quantum behavior of the simple pendulum. A second possible direction of research is to investigate whether or not the dynamics of the pendulum could be used also to implement one-qubit quantum gates as the rigid body does [37].

\section{References}

[1] Jacobi, C. G. J., Fundamenta nova theoriae functionum ellipticarum, Königsberg: Borntraeger, 1829.

[2] Whittaker, E. T., A Treatise on the Analytical Dynamics of Particles and Rigid Bodies, 4th ed., New York: Cambridge Univ. Press, 1989.

[3] Du Val, P., Elliptic Functions and Elliptic Curves, London Math. Soc. Lecture Note Ser., vol.9, Cambridge: Cambridge Univ. Press, 1973.

[4] Lang, S., Elliptic Functions, Grad. Texts in Math., vol.112, New York: Springer, 1973.

[5] Lawden, D.F., Elliptic Functions and Applicaions, Appl. Math. Sci., vol.80, New York: Springer, 1989.

[6] McKean, H. and Moll, V., Elliptic Curves: Function Theory, Geometry, Arithmetic, Cambridge: Cambridge Univ. Press, 1999.

[7] Armitage, J. V. and Eberlein, W. F., Elliptic Functions, London Math. Soc. Stud. Texts, vol. 67, Cambridge: Cambridge Univ. Press, 2006.

RUSSIAN JOURNAL OF NONLINEAR DYNAMICS, 2020, 16(1), 133-159 
[8] Poinsot, L., Outlines of a New Theory of Rotatory Motion, Cambridge: Cambridge Univ. Press, 1834.

[9] Landau, L. D. and Lifshitz, E. M., Course of Theoretical Physics: Vol. 1. Mechanics, 3rd ed., Oxford: Pergamon, 1976.

[10] Marsden, J.E. and Ratiu, T.S., Introduction to Mechanics and Symmetry: A Basic Exposition of Classical Mechanical Systems, Texts Appl. Math., vol. 17, New York: Springer, 1994.

[11] Piña Garza, E., Dinámica de Rotaciones, Colección CBI, México: Univ. Autónoma Metropolitana, 1996.

[12] Holm, D. D., Geometric Mechanics: P. 1. Dynamics and Symmetry, 2nd rev. ed., Singapore: World Sci., 2011.

[13] Appell, P., Principes de la théorie des fonctions elliptiques et applications, Paris: Gauthier-Villars, 1897.

[14] von Helmholtz, H., Vorlesungen über die Dynamik discreter Massenpunkte, Leipzig: Barth, 1898.

[15] Méndez-Fragoso, R. and Ley-Koo, E., Angular Momentum Theory in Bases of Lamé Spheroconal Harmonics, Adv. Quantum Chem., 2015, vol.71, pp. 115-152.

[16] Abel, N. H., Recherches sur les fonctions élliptiques, J. Reine Angew. Math., 1827, vol. 2, pp. $101-181$.

[17] Jacobi, C. G. J., Demonstratio theorematis ad theoriam functionum ellipticarum spectantis, Astron. Nachr., 1827, vol. 6, no. 10, pp. 133-141.

[18] Beléndez, A., Pascual, C., Méndez, D. I., Beléndez, T., and Neipp, C., Exact Solution for the Nonlinear Pendulum, Rev. Bras. Ensino Fís., 2007, vol. 29, no. 4, pp. 645-648.

[19] Ochs, K., A Comprehensive Analytical Solution of the Nonlinear Pendulum, Eur. J. Phys., 2011, vol. 32, no. 2, pp. 479-490.

[20] Linares, R., Duality Symmetries behind Solutions of the Classical Simple Pendulum, Rev. Mex. Fís. E, 2018, vol. 64, no. 2, pp. 205-221.

[21] Condon, E. U., The Physical Pendulum in Quantum Mechanics, Phys. Rev., 1928, vol.31, no.5, pp. 891-894.

[22] Pradhan, T. and Khare, A. V., Plane Pendulum in Quantum Mechanics, Am. J. Phys., 1973, vol.41, no. 1, pp. 59-66.

[23] Aldrovandi, R. and Leal Ferreira, P., Quantum Pendulum, Am. J. Phys., 1980, vol.48, no.8, pp. 660-664.

[24] Euler, L., Du mouvement de rotation des corps solides autour d'un axe variable, Mémoires de l'académie des sciences de Berlin, 1765, vol.14, pp.154-193; see also: Opera Omnia, Ser. 2: Vol.8, pp. 200-235.

[25] Kramers, H. A. and Ittmann, G. P., Zur Quantelung des asymmetrischen Kreisels, Z. Phys., 1929, vol. 53, pp. 553-565.

[26] King, G. W., The Asymmetric Rotor: 6. Calculation of Higher Energy Levels by Means of the Correspondence Principle, J. Chem. Phys., 1947, vol. 15, no. 11, pp. 820-830.

[27] Spence, R. D., Angular Momentum in Sphero-Conal Coordinates, Am. J. Phys., 1959, vol. 27, no. 5, pp. 329-335.

[28] Lukac, I. and Smorodinskii, Ya. A., The Wave Functions of an Asymmetric Top, JETP, 1970, vol. 30, no. 4, pp. 728-730; see also: Zh. Ėksper. Teoret. Fiz., 1970, vol. 57, no.4, pp. 1342-1348.

[29] Patera, J. and Winternitz, P., A New Basis for the Representation of the Rotation Group. Lamé and Heun Polinomials, J. Math. Phys., 1973, vol. 14, no. 8, pp. 1130-1139.

[30] Piña, E., Some Properties of the Spectra of Asymmetric Molecules, J. Mol. Struc. THEOCHEM, 1999, vol. 493, nos. 1-3, pp. 159-170.

[31] Valdés, M. T. and Piña, E., The Rotational Spectra of the Most Asymmetric Molecules, Rev. Mex. Fús., 2006, vol. 52, pp. 220-229.

[32] Méndez-Fragoso, R. and Ley-Koo, E., Rotations of Asymmetric Molecules and the Hydrogen Atom in Free and Confined Configurations, Adv. Quantum Chem., 2011, vol.62, pp. 137-213. 
[33] Nambu, Y., Generalized Hamiltonian Dynamics, Phys. Rev. D (3), 1973, vol. 7, pp. 2405-2412.

[34] Deprit, A., Free Rotation of a Rigid Body Studied in the Phase Plane, Amer. J. Phys., 1967, vol. 35, no. 5, pp. 424-428.

[35] Holm, D. D. and Marsden, J.E., The Rotor and the Pendulum, in Symplectic Geometry and Mathematical Physics, Progr. Math., vol. 99, Boston, Mass.: Birkhäuser, 1991, pp. 189-203.

[36] Montgomery, R., How Much Does the Rigid Body Rotate? A Berry's Phase from the 18th Century, Am. J. Phys., 1991, vol. 59, no. 5, pp. 394-398.

[37] Van Damme, L., Leiner, D., Mardešić, P., Glaser, S. J., and Sugny, D., Linking the Rotation of a Rigid Body to the Schrödinger Equation: The Quantum Tennis Racket Effect and Beyond, Sci. Rep., 2017, vol. 7, no. 1, 14 pp.

[38] Iwai, T. and Tarama, D., Classical and Quantum Dynamics for an Extended Free Rigid Body, Differential Geom. Appl., 2010, vol.28, no. 5, pp. 501-517.

[39] de la Cruz, M., Gaspar, N., Jiménez-Lara, L., and Linares, R., Classification of the Classical $S L(2, \mathbb{R})$ Gauge Transformations in the Rigid Body, Ann. Physics, 2017, vol.379, pp. 112-130.

[40] Brizard, A.J., A Primer on Elliptic Functions with Applications in Classical Mechanics, Eur. J. Phys., 2009, vol. 30, no. 4, pp. 729-750.

[41] Appell, P., Sur une interprétation des valeurs imaginaires du temps en Mécanique, Comptes rendus hebdomadaires des séances de l'Académie des sciences, 1878, vol. 87, pp. 1074-1077.

[42] David, D. and Holm, D. D., Multiple Lie-Poisson Structures, Reductions, and Geometric Phases for the Maxwell-Bloch Travelling Wave Equations, J. Nonlinear Sci., 1992, vol. 2, no. 2, pp. 241-262. 